\title{
Robust Model Predictive Control With Integral Sliding Mode in Continuous-Time Sampled-Data Nonlinear Systems
}

\author{
Matteo Rubagotti, Student Member, IEEE, Davide Martino Raimondo, Antonella Ferrara, Senior Member, IEEE, \\ and Lalo Magni
}

\begin{abstract}
This paper proposes a control strategy for nonlinear constrained continuous-time uncertain systems which combines robust model predictive control (MPC) with sliding mode control (SMC). In particular, the so-called Integral SMC approach is used to produce a control action aimed to reduce the difference between the nominal predicted dynamics of the closed-loop system and the actual one. In this way, the MPC strategy can be designed on a system with a reduced uncertainty. In order to prove the stability of the overall control scheme, some general regional input-to-state practical stability results for continuous-time systems are proved.
\end{abstract}

Index Terms-Constrained control, nonlinear predictive control (NPC), sampled data control, sliding mode control (SMC), stability of nonlinear systems.

\section{INTRODUCTION}

$\mathbf{M}$ ODEL predictive control (MPC) is a control technique which permits to cope with a constrained system providing an optimal control strategy. MPC has been widely used in the process industry and also studied in its theoretical aspects by the research community (see for an overview, [1]-[3], and the books [4]-[7]). In the last years, one of the topics of interest in MPC has been the definition of robust strategies, in order to guarantee certain stability properties and the respect of the constraints also in the presence of uncertainties and external disturbances. Several nonlinear MPC (NMPC) algorithms with guaranteed robust stability with respect to different classes of disturbances have been proposed (see, e.g., [8] and the references therein). In order to obtain a robust controller in presence of constraints two main approaches have been proposed in the literature.

- The min-max approach, where the objective function is minimized for the worst possible uncertainty realization,

Manuscript received September 21, 2009; revised February 12, 2010; accepted June 22, 2010. Date of publication September 13, 2010; date of current version March 09, 2011. This work was supported in part by the European Commission under the Project Feednetback FP7-ICT-223866 and by the Italian PRIN project "Model predictive control algorithms for artificial pancreas." Recommended by Associate Editor D. Angeli.

M. Rubagotti is with the Department of Mechanical and Structural Engineering, University of Trento, Trento 38123, Italy (e-mail: matteo. rubagotti@ing.unitn.it).

D. M. Raimondo was with the Automatic Control Laboratory, Swiss Federal Institute of Technology (ETH), Zürich 8092, Switzerland and is now with the Department of Computer Engineering and systems Science, University of Pavia, Pavia 27100, Italy (e-mail: davide.raimondo@unipv.it).

A. Ferrara and L. Magni are with the Department of Computer Engineering and Systems Science, University of Pavia, Pavia 27100, Italy (e-mail: antonella. ferrara@unipv.it; lalo.magni@unipv.it).

Digital Object Identifier 10.1109/TAC.2010.2074590

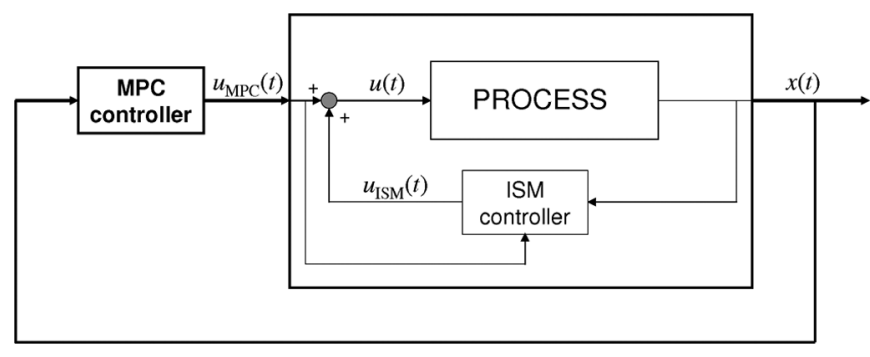

Fig. 1. Scheme of the overall hierarchical control system.

forcing the satisfaction of the constraints for any possible perturbation [9]-[14]. This approach presents a very high computational burden, and, at the moment, can be applied to systems with small size or slow dynamics.

- The open-loop nominal approach with tightened constraints, where the real constraints are shrunk in order to guarantee that the original constraints are fulfilled by the real system for any possible uncertainty realization [15]-[19]. This approach has the drawback of being too conservative in view of the open-loop off-line approximation of the worst possible effect of the disturbance that is required in order to compute the tightened constraints.

Exploiting the idea proposed in [16], the second approach is here followed. The hierarchical control scheme (see Fig. 1), composed by a NMPC algorithm and a Sliding Mode controller, is introduced in order to improve the robustness features of the NMPC algorithm. Sliding Mode Control (SMC) [20], [21] is a well-known robust control technique for nonlinear systems which guarantees the complete elimination of the effect of "matched disturbances" (i.e. disturbances acting on the control input channel) once the system is in the so-called "sliding mode," that is, the state has reached a suitable subspace of the state space, called "sliding manifold." Considering that matched disturbances are very common and can represent both parametric uncertainties and external disturbances, robustness with respect to them is a significant benefit, also taking into account that SMC requires a very low computational burden and guarantees the finite-time convergence to the sliding manifold, upon which the controlled system exhibits the desired dynamics.

Different approaches to merge MPC and Sliding Mode techniques have already been proposed in the literature. For instance, in [22] a combined scheme was presented for linear systems in the framework of Generalized Predictive Control. Another approach has been proposed in [23], where MPC was 
used to update the parameters of the sliding manifold. The Sliding Mode strategy considered in this paper is instead designed according to the so-called Integral Sliding Mode (ISM) approach [24]-[27]. This technique is a recent development of the more classical SMC design methodology that presents the advantage of forcing the system state to lie on the sliding manifold from the initial time instant. Relying on the knowledge of the nominal model of the system and of the control signal generated by the NMPC, the ISM controller is designed to produce a continuous-time control action aimed to reduce the difference between the dynamics of the nominal closed-loop system and the actual evolution of the state. In this way the NMPC can be designed on a system with reduced uncertainty, limiting the conservativeness of the open-loop nominal approach. In particular, if only matched disturbances affect the system, no tightened constraints are required in the NMPC formulation, while, if also unmatched disturbances are considered, then less conservative constraints must be introduced with respect to the pure NMPC control scheme.

The continuous-time setting is the most appropriate if the plant model is derived from first principle continuous-time equations. Nonetheless, since solving an optimization problem in continuous time would be computationally untractable, some MPC algorithms which use continuous-time models with sampled data systems have been proposed [28]-[30]. In this paper, following the approach analyzed in [29] for systems not affected by uncertainty, the optimization is performed in discrete-time with respect to piecewise-constant control signals. The continuous-time control law of the ISM is then added to the piecewise-constant signal generated by the NMPC. In order to prove the stability of the overall control scheme, the regional Input-to-State Stability (ISS) and Input-to-State practical Stability (ISpS) results introduced respectively in [31] and [32] for discrete-time systems are proved for continuous-time systems.

A preliminary version of the theoretical development here proposed, without mathematical proofs, can be found in [33]. The organization of the paper is the following: the notations used in the paper are reported in Section II, while Section III deals with the description of the system. In Section IV the overall control strategy, including the robust NMPC control strategy and the ISM controller is presented. Section V studies the stability properties of the control system. The simulation results and the conclusions are reported in Sections VI and VII, respectively. Finally, for the readers' convenience, all the proofs are in the Appendix, together with the introduction of the concept of regional ISpS in continuous-time.

\section{NOTATIONS}

The Euclidean norm is denoted as $|\cdot|$. For any symmetric matrix $A, \lambda_{\max }(A)$ and $\lambda_{\min }(A)$ denote the largest and the smallest eigenvalue of matrix $A$, respectively. Given a signal $w$, let $\mathbf{w}_{\left[t_{1}, t_{2}\right]}$ be a signal defined from time $t_{1}$ to time $t_{2}$. In order to simplify the notation, when it is inferrable from the context, the subscript of the sequence is omitted. The set of signals $w$, the values of which belong to a compact set $\mathcal{W} \subseteq \mathbb{R}^{m}$ is denoted by $\mathcal{M}_{\mathcal{W}}$, while $\mathcal{W}^{\text {sup }} \triangleq \sup _{w \in \mathcal{W}}\{|w|\}$. Moreover $\|\mathbf{w}\| \triangleq \sup _{t>0}\{|w(t)|\}$ where $w(t)$ denotes the values that the signal $w$ takes in correspondence to the time $t$. The symbol id represents the identity function from $\mathbb{R}$ to $\mathbb{R}$. Given a set $\mathcal{A} \subseteq \mathbb{R}^{n},|\zeta|_{\mathcal{A}} \triangleq \inf \{|\eta-\zeta|, \eta \in \mathcal{A}\}$ is the point-to-set distance from $\zeta \in \mathbb{R}^{n}$ to $\mathcal{A}$ while $\partial \mathcal{A}$ denotes the boundary of $\mathcal{A}$. Given two sets $\mathcal{A} \in \mathbb{R}^{n}, \mathcal{B} \in \mathbb{R}^{n}$, then the Pontryagin difference set $\mathcal{C}$ is defined as $\mathcal{C}=\mathcal{A} \sim \mathcal{B} \triangleq\left\{x \in \mathbb{R}^{n}: x+\xi \in \mathcal{A}, \forall \xi \in \mathcal{B}\right\}$ The floor function $\lfloor\cdot\rfloor$ is defined as follows: $\lfloor x\rfloor \triangleq \max \{n \in$ $\mathbb{Z}: n \leq x\}$. A function $\gamma: \mathbb{R}_{\geq_{0}} \rightarrow \mathbb{R}_{\geq_{0}}$ is of class $\mathcal{K}$ (or a " $\mathcal{K}$-function") if it is continuous, positive definite and strictly increasing, and $\gamma(0)=0$. A function $\gamma: \mathbb{R}_{\geq_{0}} \rightarrow \mathbb{R}_{\geq_{0}}$ is of class $\mathcal{K}_{\infty}$ if it is a $\mathcal{K}$-function and $\gamma(s) \rightarrow+\infty$ as $s \rightarrow+\infty$. A function $\beta: \mathbb{R}_{\geq_{0}} \times \mathbb{Z}_{\geq_{0}} \rightarrow \mathbb{R}_{\geq_{0}}$ is of class $\mathcal{K} \mathcal{L}$ if, for each fixed $t \geq 0, \bar{\beta}(\cdot, t)$ is of class $\mathcal{K}$, for each fixed $s \geq 0$, $\beta(s, \cdot)$ is decreasing and $\beta(s, t) \rightarrow 0$ as $t \rightarrow \infty$. Given a matrix $M \in \mathbb{R}^{n \times m}$ with $n>m$, then its orthogonal complement is $M^{\perp} \in \mathbb{R}^{n \times(n-m)}$.

\section{Problem Statement}

In this paper, it is assumed that the plant to be controlled is described by the continuous-time nonlinear model

$$
\dot{x}(t)=\mathcal{F}(x(t), u(t), d(t)), \quad t \geq 0
$$

where $x \in \mathbb{R}^{n}$ is the state, $u \in \mathcal{U}$ is the current control vector, $d \in \mathbb{R}^{q}$ is the disturbance term, and $\mathcal{U} \subset \mathbb{R}^{m}$ is a compact set containing the origin as an interior point. Given system (1), which is assumed to be forward complete, assume also that $f: \mathbb{R}^{n} \times \mathbb{R}^{m} \rightarrow \mathbb{R}^{n}$ denotes the nominal model, being $f(x(t), u(t)) \triangleq h(x(t))+B u(t)$, with $h: \mathbb{R}^{n} \rightarrow \mathbb{R}^{n}$, $B \in \mathbb{R}^{m \times n}, \operatorname{rank}(B)=m$. The system can then be expressed as

$$
\dot{x}(t)=h(x(t))+B u(t)+w(t), \quad t \geq 0
$$

where $w \in \mathbb{R}^{n}$ and $w \triangleq \mathcal{F}(x(t), u(t), d(t))-f(x(t), u(t))$ denotes the additive uncertainty. The solution of system (2) with initial state $x(0)=\bar{x}$ and the uncertain signal $\mathbf{w}$ is denoted by $\varphi(t, \bar{x}, \mathbf{u}, \mathbf{w})$.

Remark 1: The control-affine form $f(x(t), u(t))=$ $h(x(t))+B u(t)$ is required in order to obtain an explicit control law for the ISM strategy that will be presented in the sequel.

System (2) is supposed to fulfill the following assumption.

Assumption 1:

1) System (2) is forward complete.

2) $h(0)=0$.

3) The state and control variables are restricted to fulfill the following constraints

$$
\begin{aligned}
& x \in \mathcal{X} \\
& u \in \overline{\mathcal{U}}
\end{aligned}
$$

where $\mathcal{X}$ and $\overline{\mathcal{U}} \subseteq \mathcal{U}$ are compact sets containing the origin as an interior point.

4) The uncertainty $w$ is such that 
where $\mathcal{W}$ is a compact set containing the origin, with $\mathcal{W}^{\text {sup }}$ known.

Remark 2: This last property can be obtained starting from system (1), if it is assumed that the disturbance $d$ is such that $d \in \mathcal{D}$, where $\mathcal{D}$ is a compact set containing the origin, with $\mathcal{D}^{\text {sup }}$ known. Given (3), (4), one can state that $w$ can be modeled as a bounded uncertainty as well.

Given a suitable sampling period $T$, and letting $t_{k}$, with $k \geq$ 0 , be the sampling instants, the control objective consists in designing a piecewise-constant control law that steers the system state to (a neighborhood of) the origin fulfilling the constraints on the input and the state itself along the system evolution for any possible uncertainty satisfying Point 4 in Assumption 1, and yielding an optimal closed-loop performance according to a certain performance index.

Assumption 2: System (2) is defined such that, considering a generic time instant $\tilde{t} \triangleq t_{k}+\tau, 0 \leq \tau \leq T$,

1) given two different initial conditions $x_{1}$ and $x_{2} \in \mathcal{X}$ at time 0 , and a signal $\mathbf{u} \in \mathcal{M}_{\mathcal{U}}$, it yields

$$
\left|\varphi\left(\tilde{t}, x_{1}, \mathbf{u}, \mathbf{0}\right)-\varphi\left(\tilde{t}, x_{2}, \mathbf{u}, \mathbf{0}\right)\right| \leq \mathcal{L}_{\tau} \mathcal{L}_{T}^{k}\left|x_{1}-x_{2}\right|
$$

where $\mathcal{L}_{\tau} \triangleq \mathcal{L}(\tau)$ is a positive continuous function defined in $[0 ; T]$, such that $\mathcal{L}_{0}=1$. The term $\mathcal{L}_{T}^{k}=\left(\mathcal{L}_{T}\right)^{k}$ stands for $\mathcal{L}_{T}$ raised to the $k$-th power.

2) given an initial condition $\bar{x}$ at time 0 , the signals $\mathbf{u} \in \mathcal{M}_{\mathcal{U}}$ and $\mathbf{w} \in \mathcal{M}_{\mathcal{W}}$, one has that

$$
|\varphi(\tau, \bar{x}, \mathbf{u}, \mathbf{0})-\varphi(\tau, \bar{x}, \mathbf{u}, \mathbf{w})| \leq \gamma \tau
$$

where $\gamma \in \mathbb{R}_{\geq 0}$ is a constant value and $\bar{x} \in \mathcal{X}$.

In order to evaluate the discrepancy between the nominal and perturbed evolutions of the system at a generic time instant, the following lemma can be stated.

Lemma 1: Suppose that Assumptions 1 and 2 are satisfied. Then, given $\tilde{t} \triangleq t_{k}+\tau$ and $x(0)=\bar{x}$, one has that

$$
|\varphi(\tilde{t}, \bar{x}, \mathbf{u}, \mathbf{0})-\varphi(\tilde{t}, \bar{x}, \mathbf{u}, \mathbf{w})| \leq \gamma\left(\tau+T \mathcal{L}_{\tau} \frac{\mathcal{L}_{T}^{k}-1}{\mathcal{L}_{T}-1}\right)
$$

for all $\bar{x} \in \mathcal{X}$, all $\mathbf{u} \in \mathcal{M}_{\mathcal{U}}$ and $\mathbf{w} \in \mathcal{M}_{\mathcal{W}}$.

Proof: See Appendix C.

Given the feedback control law

$$
u(t)=\kappa\left(x\left(t_{k}\right)\right), \quad t \in\left[t_{k}, t_{k+1}\right)
$$

following the idea used in [29], the description of the hold mechanism implicit in (8) calls for a state augmentation. Letting $x_{c} \triangleq$ $\left[\begin{array}{ll}x^{T} & u^{T}\end{array}\right]^{T} \in \mathbb{R}^{n+m}$, the closed loop system (2), (8) is given by

$$
\begin{aligned}
\dot{x}_{c}(t) & =\left[\begin{array}{l}
h(x(t)+B u(t)+w(t) \\
0_{m, 1}
\end{array}\right], \quad t \in\left[t_{k}, t_{k+1}\right) \\
x_{c}\left(t_{k}\right) & =\left[\begin{array}{l}
x\left(t_{k}^{-}\right) \\
\kappa\left(x\left(t_{k}^{-}\right)\right)
\end{array}\right]
\end{aligned}
$$

and its solution from initial time 0 and initial state $x_{c}(0)=\bar{x}_{c}$ is given by $\varphi_{c}\left(t, \bar{x}_{c}, \mathbf{w}\right)$. Moreover, the first $n$ and the last $m$ components of $\varphi_{c}\left(t, \bar{x}_{c}, \mathbf{w}\right)$ will be denoted by $\varphi_{x}\left(t, \bar{x}_{c}, \mathbf{w}\right)$ and $\varphi_{u}\left(t, \bar{x}_{c}, \mathbf{w}\right)$, respectively.

\section{RobUST NMPC WITH ISM: THE OVERALl CONTROL STRATEGY}

Robust NMPC with tightened constraints can lead to very conservative solutions or even to unfeasible problems. In order to mitigate the conservativeness of the approach, the hierarchical control scheme represented in Fig. 1 is here proposed, where the ISM controller is introduced in order to reduce the uncertainty that must be taken into account by the NMPC controller.

The control variable $u(t)$, according to Fig. 1, is composed by two parts, i.e.

$$
u(t)=u_{\mathrm{MPC}}(t)+u_{\mathrm{ISM}}(t)
$$

where the component $u_{\mathrm{MPC}}(t)$ is generated by the NMPC controller, while $u_{\mathrm{ISM}}(t)$ is generated by the ISM controller to compensate for the uncertain terms. As a cost to pay for the uncertainty reduction, one has that part of the control effort must be used by the ISM controller so that the input constraints imposed in the NMPC optimization problem are smaller than the actuators limits. The ISM controller, based on the knowledge of the nominal continuous-time model of the system and of the control signal generated by the NMPC control law, produces a control action in order to reduce the difference between the dynamics of the nominal closed-loop system and the actual evolution of the state. Moreover, the ISM, having a negligible computational burden, can be used in continuous-time.

\section{A. Integral Sliding Mode Strategy}

Given system (2), define a sliding manifold, i.e. a set $\mathcal{S}=$ $\{x \in \mathcal{X}: s(x, t)=0\}$, where, as in [34]

$s(x(t), t) \triangleq G\left[x(t)-x(0)-\int_{0}^{t} h(x(\tau))+B u_{\mathrm{MPC}}(\tau) d \tau\right]$

and $G \in \mathbb{R}^{m \times n}$ is a projection matrix, defined such that the matrix product $G B$ is invertible. It is important to note that the initial state belongs to the sliding manifold, i.e. $s(x(0), 0)=$ 0 . The uncertain term can always be seen as the sum of two different parts

$$
w(t)=B w_{\mathrm{M}}(t)+B^{\perp} w_{\mathrm{U}}(t)
$$

where $B^{\perp} \in \mathbb{R}^{m \times(n-m)}, w_{\mathrm{M}}(t) \in \mathbb{R}^{m}$, and $w_{\mathrm{U}}(t) \in \mathbb{R}^{n-m}$. The uncertainty $B w_{\mathrm{M}}(t)$ is the matched uncertainty, and can be perfectly compensated by the SMC action [20]. The other term is called "unmatched uncertainty" and cannot be compensated by a sliding mode strategy. The control variable $u_{\mathrm{ISM}}(t)$ can be defined in several ways, for instance by relying on to the so-called unit-vector approach

$$
u_{\mathrm{ISM}}(t) \triangleq-\rho \frac{(G B)^{T} s(x(t), t)}{\left|(G B)^{T} s(x(t), t)\right|}
$$


where $\rho$ is the controller gain (considered constant here for the sake of simplicity) large enough to keep $s(x(t), t)=0$ from the initial time instant. The choice of this particular gain as $\rho=\mathcal{W}^{\text {sup }}$ will permit to dominate the matched uncertainty term: in principle, one could also choose a larger value for $\rho$, but this would induce useless wear in the system and would subtract some usable control amplitude to the NMPC controller. However, some variations of this technique, e.g. letting $\rho$ be a function of the states and/or a time-varying value can be found in the literature (see, e.g. [21]). To determine the motion equations at the sliding manifold, the equivalent control method [20] is used. The equivalent control is the continuous signal which can be determined by solving, with respect to $u_{\mathrm{ISM}}(t)$, the equation $\dot{s}(x(t), t)=0$, taking into account (2) and (10). Substituting the value of the equivalent control in (2), it yields

$$
\dot{x}(t)=h(x(t))+B u_{\mathrm{MPC}}(t)+\tilde{w}(t)
$$

where

$$
\tilde{w}(t)=\left(I-B(G B)^{-1} G\right) B^{\perp} w_{\mathrm{U}}(t)
$$

Note that the perturbation $B w_{\mathrm{M}}(t)+B^{\perp} w_{\mathrm{U}}(t)$ has been replaced by $\tilde{w}(t)$. So, the amount of reduction of the uncertain term depends on the choice of $G$.

Lemma 2: Applying to system (2) the control law (11) based on the sliding manifold (10), with $G=B^{T}$, one has that the choice $G=B^{T}$ minimizes the norm of $\tilde{w}(t)$, i.e.

$$
B^{T}=\arg \min _{G \in \mathbb{R}^{m \times n}}\left|\left(I-B(G B)^{-1} G\right) B^{\perp} w_{\mathrm{U}}(t)\right| .
$$

Then, the resulting system that the NMPC controller has to consider is

$$
\dot{x}(t)=h(x(t))+B u_{\mathrm{MPC}}(t)+B^{\perp} w_{\mathrm{U}}(t)
$$

where the matched uncertainty has been eliminated, while the unmatched one has not changed.

Proof: See Propositions 2 and 3 in [27].

For each component of the control variable $u(i)=$ $u_{\mathrm{MPC}}(i)+u_{\mathrm{ISM}}(i), i=1, \ldots, m$, given the approach selected to design the ISM component, the value of the amplitude of the terms $u_{\mathrm{ISM}}(i)$ can be computed, and then a new set $\overline{\mathcal{U}}^{R}$ can be accordingly found as

$$
\overline{\mathcal{U}}^{R}=\left\{u_{\mathrm{MPC}}: u_{\mathrm{MPC}} \in \overline{\mathcal{U}}^{R} \Rightarrow u \in \mathcal{U}\right\} .
$$

Defining $u_{\mathrm{ISM}}(t)$ as in (11), a quantity equal to $\rho$ must be subtracted to each component of the control bounds for the NMPC controller.

Remark 3: It is very important to note that it would be possible to use the ISM strategy here described even if the term $B$ were state dependent; nonetheless, no solution has been proposed up to now in the literature to find the optimal realization of the sliding manifold that can avoid any increasing of the unmatched uncertainty. So, in case $B=B(x)$, depending on the specific system, one would have that the elimination of the matched uncertainty could imply an increasing of the unmatched one, which must be kept as small as possible with the choice of a suitable sliding manifold.

Remark 4: In case no unmatched uncertainties are present (i.e. $n=m$, or $w_{\mathrm{U}}(t)=0, \forall t>0$ ), then this strategy will completely eliminate the uncertain terms, so that the NMPC controller has to control a system without uncertain terms. If this is the case, a robust NMPC strategy is no longer needed, and one can use the technique described in [29].

\section{B. Robust NMPC Strategy}

The robust NMPC controller must be designed for system (12) formed by the system under control complemented with the ISM control law. Following the idea behind the control algorithm presented in [16] for discrete-time systems and considering that system (12) is a particular case of system (2), a new robust NMPC control algorithm for continuous-time systems in form (2) is described in this subsection. Some preliminary definitions and results are first introduced. In particular, in order to describe a key ingredient of the robust NMPC controller, i.e. the tightened constraints, define the tightened set

$$
\mathcal{X}_{k T+\tau}=\mathcal{X} \sim \mathcal{B}_{k T+\tau}
$$

where

$$
\mathcal{B}_{k T+\tau} \triangleq\left\{z \in \mathbb{R}^{n}:|z| \leq \gamma\left(\tau+T \mathcal{L}_{\tau} \frac{\mathcal{L}_{T}^{k}-1}{\mathcal{L}_{T}-1}\right)\right\} .
$$

This definition of the tightened set guarantees that, if the nominal state evolution belongs to $\mathcal{X}_{k T+\tau}$ in (14), then the perturbed trajectory of the system fulfills (3), as will be proved in the sequel.

The proposed NMPC controller is based on the following Finite-Horizon Optimal Control Problem (FHOCP) that consists in minimizing, at any sampling time instant $t_{k}$, a suitable cost function with respect to the control sequence $\overline{\mathbf{u}}_{\left[t_{k}, t_{k+N-1} \mid t_{k}\right]} \triangleq$ $\left[u_{0}\left(t_{k}\right), u_{1}\left(t_{k}\right), \ldots, u_{N-1}\left(t_{k}\right)\right]$, with $N \geq 1$ being the prediction horizon. The associated finite horizon piecewise-constant control signal $\mathbf{u}_{\left[t_{k}, t_{k+N} \mid t_{k}\right)}(t)$ is such that

$$
\mathbf{u}_{\left[t_{k}, t_{k+N} \mid t_{k}\right)}\left(t \mid t_{k}\right)=u_{j}\left(t_{k}\right)
$$

for all $t \in\left[t_{k+j}, t_{k+j+1}\right)$ and all $j \in[0, \ldots, N-1]$.

Definition 1 (FHOCP): Consider system (2) with $x\left(t_{k}\right)=\bar{x}$. Given the positive integer $N$, the quadratic stage cost $l(x, u) \triangleq x^{T} Q x+u^{T} R u$ ( $Q$ and $R$ being positive definite matrices), the quadratic terminal penalty $V_{f}(x) \triangleq x^{T} \Pi x$ (being $\Pi$ a symmetric positive definite matrix) and the terminal set $\mathcal{X}_{f}$, the FHOCP problem consists in minimizing with respect to $\overline{\mathbf{u}}_{\left[t_{k}, t_{k+N-1} \mid t_{k}\right]}$ the cost function

$$
\begin{aligned}
J\left(\bar{x}, \overline{\mathbf{u}}_{\left[t_{k}, t_{k+N-1} \mid t_{k}\right]}, N\right) & \\
& =\int_{t_{k}}^{t_{k+N}} l(x(\tau), u(\tau)) d \tau+V_{f}\left(x\left(t_{k+N}\right)\right)
\end{aligned}
$$


subject to

1) the state dynamics (2) with $w(t)=0$, for all $t \in\left[t_{k}, t_{k+N}\right)$

2) the state constraint $x(t) \in \mathcal{X}_{t-t_{k}}$, for all $t \in\left[t_{k}, t_{k+N}\right]$;

3) the control constraint (4);

4) the terminal state constraint $x\left(t_{k+N}\right) \in \mathcal{X}_{f}$.

Remark 5: Since $\mathcal{X}$ and $\overline{\mathcal{U}}$ are bounded, the stage cost is a Lipschitz function with respect to both the state and the control values, i.e., there exist $\mathcal{L}_{l}>0$ and $\mathcal{L}_{l u}>0$ such that

$$
\begin{aligned}
& \left|l\left(x_{1}, u\right)-l\left(x_{2}, u\right)\right| \leq \mathcal{L}_{l}\left|x_{1}-x_{2}\right| \\
& \left|l\left(x, u_{1}\right)-l\left(x, u_{2}\right)\right| \leq \mathcal{L}_{l u}\left|u_{1}-u_{2}\right|
\end{aligned}
$$

for all $u_{1}, u_{2} \in \overline{\mathcal{U}}$ and all $x_{1}, x_{2} \in \mathcal{X}$.

It is now possible to define the NMPC algorithm: at any sampling time instant $t_{k}$, find the optimal control sequence $\overline{\mathbf{u}}_{\left[t_{k}, t_{k+N-1} \mid t_{k}\right]}^{o}$ by solving the FHOCP. Then, according to the Receding Horizon strategy, define $\kappa^{M P C}\left(x\left(t_{k}\right)\right) \triangleq \overline{\mathbf{u}}_{\left[t_{k}, t_{k+N-1} \mid t_{k}\right]}^{o}\left(t_{k}\right)$, and apply the control law

$$
u(t)=\kappa^{M P C}\left(x\left(t_{k}\right)\right), \quad t \in\left[t_{k}, t_{k+1}\right) .
$$

The following assumption on the choice of the design parameters is introduced.

Assumption 3: The design parameters $V_{f}$ and $\mathcal{X}_{f}$ are such that, given a compact set $\Phi$ and an auxiliary control law $\kappa_{f}$,

1) $\mathcal{X}_{f}(x) \triangleq\left\{x: x^{T} \Pi x \leq \sigma_{f}\right\}, \mathcal{X}_{f} \subseteq \mathcal{X}$, such that $\{0\} \subseteq$ $\mathcal{X}_{f}$ and $\sigma_{f}$ is a positive real number;

2) $\Phi \triangleq\left\{x: x^{T} \Pi x \leq \sigma_{\Phi}\right\}$, being $\mathcal{X}_{f} \subseteq \Phi \subseteq \mathcal{X}_{(N-1) T}$, and $\sigma_{\Phi} \geq \sigma_{f}$

3) $\kappa_{f}(x(t)) \triangleq \kappa_{f}\left(x\left(t_{k}\right)\right)$, for all $t \in\left[t_{k}, t_{k+1}\right)$, with $x\left(t_{k}\right)=$ $0 \Rightarrow \kappa_{f}\left(x\left(t_{k}\right)\right)=0$ and $x\left(t_{k}\right) \in \Phi \Rightarrow \kappa_{f}\left(x\left(t_{k}\right)\right) \in \overline{\mathcal{U}}$.

4) $\kappa_{f}$ is Lipschitz with respect to the state variable $x$ in the domain $\Phi$ with Lipschitz constant $\mathcal{L}_{\kappa}>0$, i.e.

$$
\left|\kappa_{f}\left(x_{1}\right)-\kappa_{f}\left(x_{2}\right)\right| \leq \mathcal{L}_{\kappa}\left|x_{1}-x_{2}\right|, \quad \forall x_{1}, x_{2} \in \Phi .
$$

5) if $x\left(t_{k}\right) \in \Phi$, then $\varphi\left(t_{k}+\tau, x\left(t_{k}\right), \kappa_{f}\left(x\left(t_{k}\right)\right), 0\right) \in$ $\mathcal{X}_{(N-1) T+\tau}$ for all $\tau \in[0, T)$. Moreover, one has that $\varphi\left(t_{k+1}, x\left(t_{k}\right), \kappa_{f}\left(x\left(t_{k}\right)\right), \mathbf{0}\right) \in \mathcal{X}_{f}$

6) the following inequality holds:

$$
\begin{aligned}
& V_{f}\left(\varphi\left(t_{k+1}, x\left(t_{k}\right), \kappa_{f}\left(x\left(t_{k}\right)\right), \mathbf{0}\right)\right)-V_{f}\left(x\left(t_{k}\right)\right) \\
& \quad \leq-\int_{t_{k}}^{t_{k+1}} l\left(\varphi\left(\tau, x\left(t_{k}\right), \kappa_{f}\left(x\left(t_{k}\right)\right), \mathbf{0}\right), \kappa_{f}\left(x\left(t_{k}\right)\right)\right) d \tau
\end{aligned}
$$

for all $x\left(t_{k}\right) \in \Phi$;

7) consider a generic time instant $t_{k}+\tau, 0 \leq \tau \leq T$; system (2) is such that

$$
\begin{aligned}
\mid \varphi\left(t_{k}+\tau, 0, \kappa_{f}\left(x\left(t_{k}\right)\right), \mathbf{0}\right)-\varphi\left(t_{k}\right. & +\tau, 0,0, \mathbf{0}) \mid \\
& \leq \mathcal{L}_{u_{\tau}}\left|\kappa_{f}\left(x\left(t_{k}\right)\right)\right|
\end{aligned}
$$

for all $x\left(t_{k}\right) \in \Phi$, where $\mathcal{L}_{u_{\tau}} \triangleq \mathcal{L}_{u}(\tau)$ is a positive continuous function defined in $[0 ; T]$ such that $\mathcal{L}_{u_{0}}=0$.
Since $\Phi$ is a compact set, no additional assumption is needed to state that $V_{f}$ is Lipschitz with respect to the state variable $x$ in the domain $\Phi$, i.e.

$$
\left|V_{f}\left(x_{1}\right)-V_{f}\left(x_{2}\right)\right| \leq \mathcal{L}_{f}\left|x_{1}-x_{2}\right|, \quad \forall x_{1}, x_{2} \in \Phi .
$$

Remark 6: In the FHOCP, continuous-time state constraints are considered. It can appear that this approach is only conceptual, because a numerical implementation would need a time discretization and the constraints satisfaction could be checked only at the integration time instants. Nonetheless, this is not a significant limitation: choosing an integration step $\tau_{i}$ small enough in the optimization phase to simulate the plant (i.e. $\tau_{i} \ll$ $T$ ), one can still have guarantees on the convergence properties of the control system without increasing the conservativeness due to the numerical approximation (see Theorem 3 in [29] for a detailed analysis).

\section{InPUT-To-State Practical Stability OF THE CLOSED-LOOP SYSTEM}

In this section, the Input-to-State practical Stability of the closed-loop system (12), (17) is proved. In order to improve the readability, the concept of continuous-time regional ISpS, that will be used along this section, is introduced and analyzed in Appendix C.

In the following, let $\mathcal{X}^{M P C}$ denote the set of states for which a solution of the FHOCP exists, and consider the following assumption.

Assumption 4: Suppose that the system parameters have been chosen obtaining a value of $\gamma$ such that

$$
\gamma \leq \frac{\sigma_{\Phi}-\sigma_{f}}{T \mathcal{L}_{f} \mathcal{L}_{T}^{N-1}}
$$

Lemma 3: [Feasibility] Suppose that system (2) satisfies Assumptions $1-4$. Then, $\mathcal{X}^{M P C}$ is a robust positively invariant set (see Definition 3 in Appendix B) for the closed-loop system (2), (17).

Proof: See Appendix C.

Lemma 4: [Regional ISpS] Suppose that system (2) fulfills Assumptions 1-4. Then, the closed-loop system (2), (17) is regional ISpS in $\mathcal{X}^{M P C}$.

Proof: See Appendix C.

Remark 7: Note that Lemmas 3 and 4 are proved for a system in the general form $\dot{x}(t)=f(x(t), u(t))+w(t)$, and then their applicability is not limited to control-affine systems.

We are now ready to introduce the main stability result for the overall control scheme.

Theorem 1: Suppose that system (2) fulfills Assumptions 1-4 with $w=B^{\perp} w_{\mathrm{U}}(t)$ and $\overline{\mathcal{U}}=\overline{\mathcal{U}}^{R}$. Then, the hierarchical closed-loop system defined by (2), (9), (11), (17) (computed with respect to system (12)) is regional ISpS in $\mathcal{X}^{M P C}$.

Proof: See Appendix C.

Note that, with respect to Lemmas 3 and 4, the assumptions must be verified with a modified disturbance $w$. This is due to the presence of the ISM inner loop that, using part of the control energy, rejects the matched disturbance. 


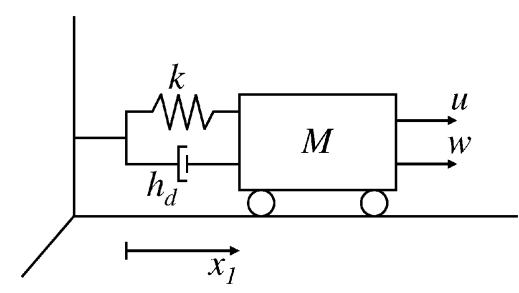

Fig. 2. Graphical representation of the simulation example.

\section{Simulation Results}

In this section, the proposed control strategy is applied to a cart moving on a plane (Fig. 2). The structure of the model is the same as in [35], and is represented by

$$
\begin{aligned}
& \dot{x}_{1}(t)=x_{2}(t)+w_{1}(t) \\
& \dot{x}_{2}(t)=\frac{1}{M}\left(-k_{0} e^{-x_{1}(t)} x_{1}(t)-h_{d} x_{2}(t)+u(t)+w_{2}(t)\right)
\end{aligned}
$$

where $x_{1}$ is the displacement of the cart with respect to the equilibrium position, and $x_{2}$ is its velocity. $M$ is the mass of the cart, $k=k_{0} e^{-x_{1}}$ is the stiffness of the spring, and $h_{d}$ is the damping factor. The control variable is the force $u$ applied to the cart, while $w_{2}$ is the load force given by the wind. The presence of another disturbance term $w_{1}$ is assumed. The values of the parameters are $M=1 \mathrm{~kg}, k_{0}=0.33 \mathrm{~N} / \mathrm{m}, h_{d}=1.1 \mathrm{Ns} / \mathrm{m}$. The uncertain terms are bounded as follows: $\left|w_{1}\right| \leq 10^{-3} \mathrm{~m} / \mathrm{s}$ and $\left|w_{2}\right| \leq 1 \mathrm{~N}$, while the control and state constraints are $|u| \leq 4 \mathrm{~N},\left|x_{1}\right| \leq 3 \mathrm{~m},\left|x_{2}\right| \leq 3 \mathrm{~m} / \mathrm{s}$, respectively. The considered system is a control-affine system with matched $\left(w_{2}\right)$ and unmatched $\left(w_{1}\right)$ disturbances. The matched one is quite common in mechanical systems, when both the control variables and the disturbances are forces or torques. The sampling time of the NMPC control law is chosen as $T=0.4 \mathrm{~s}$, while the prediction horizon is $N=3$. The matrices in the stage cost defined in the FHOCP are chosen as $Q=\rrbracket_{2}$ and $R=0.1$. As for Assumption 3 , the auxiliary control law and the matrix $\Pi$ which defines $V_{f}$ are found following the approach suggested in [29], and are equal to

$$
\kappa_{f}\left(x\left(t_{k}\right)\right)=\mathcal{K} x\left(t_{k}\right), \quad \mathcal{K}=\left[\begin{array}{ll}
-0.3151 & -1.0351
\end{array}\right]
$$

and

$$
\Pi=\left[\begin{array}{ll}
55.7749 & 15.8716 \\
15.8716 & 17.4491
\end{array}\right]
$$

respectively. The auxiliary control law is Lipschitz with respect to the state value because it is a proportional control law applied in a bounded state region.

Example 1: NMPC Only, No Matched Disturbance: Consider $w_{2}(t)=0, \forall t \geq 0$. In this case, Assumption 1 is satisfied. As for Assumption 2, the values of the parameters required for the controller design have been numerically found. They are $\gamma=3.4 \cdot 10^{-3}, \mathcal{L}_{\tau}=1+9.8 \tau, \forall \tau \in[0, T]$. With $\sigma_{f}=93$ and $\sigma_{\Phi}=108$, all the points of Assumptions 3 are satisfied. Indeed, since $\mathcal{L}_{f}=139$, one obtains that Assumption 4 is fulfilled. In Fig. 3, the evolution of the state, the control, and the unmatched disturbance is depicted, in a simulation example obtained using only the NMPC controller with initial condition $\bar{x}=[-2.2-$ 1.7]. If all the assumptions are fulfilled, it is also interesting to
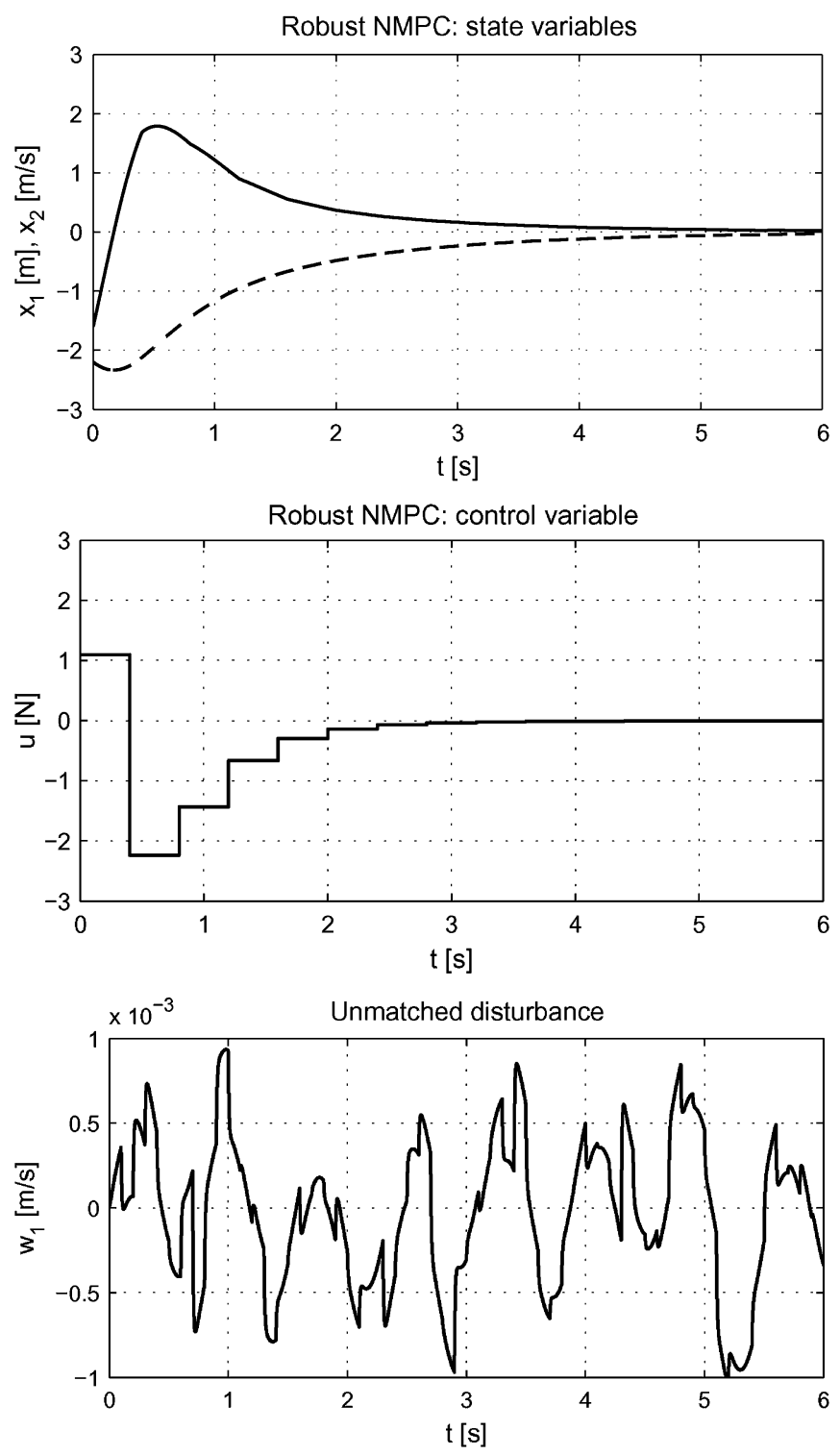

Fig. 3. Time evolution of the state variables ( $x_{1}$ : dashed line, $x_{2}$ : solid line), of the control variable and of the unmatched disturbance for the NMPC controller.

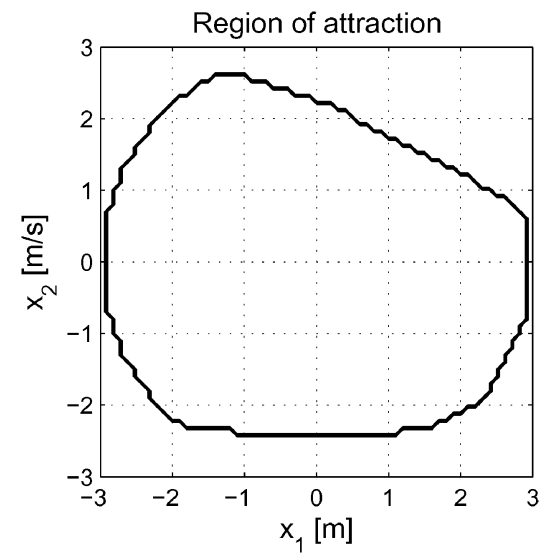

Fig. 4. Region of attraction for both Examples 1 and 3.

analyze the region of attraction, i.e. the region formed by the states for which a solution of the FHOCP exists. In Fig. 4 the region of attraction for this example is reported. 
Example 2: NMPC Only, Full Disturbance: We allow now the matched disturbance to assume values different from zero, i.e. $\left|w_{2}\right| \leq 1 \mathrm{~N}$, using again the NMPC controller only. The function $\overline{\mathcal{L}}_{\tau}$ is the same as in the previous example, while the numerical evaluation of the needed parameters leads to $\gamma=1$, $\sigma_{f}=78, \sigma_{\Phi}=89, \mathcal{L}_{f}=126$, which implies the non-fulfilment of Assumption 4, and the consequent loss of the regional ISpS property. As a practical test, we decided to perform a simulation example in the same conditions of Fig. 3, but with the increased disturbance (knowing that there is no a-priori guarantee of convergence). As a result, no feasible solution of the FHOCP can be found at the initial time instant.

Example 3: NMPC-ISM, Full Disturbance: Following the procedure of [27], the sliding manifold in (10) is chosen as $C(x)=C x$, where $C=\left[\begin{array}{ll}0 & 1\end{array}\right]$. In this way the effect of a matched disturbance $w_{2}$ is eliminated and the effect of $w_{1}$ is unchanged. A pseudo-sliding mode technique, which makes use of an approximation of the sign function [21], is used to define the ISM control variable, the maximum amplitude of which is equal to the maximum amplitude of $w_{2}$. In practice, this approximation is often used to avoid the so-called "chattering" effect (see [36]-[39], and the references therein) that is a high frequency oscillation of the sliding variable due to the discontinuous control input. In this way, the maximum control effort that must be allocated for the ISM controller is equal to the maximum amplitude of $w_{2}$ that is $1 \mathrm{~N}$ and then only $3 \mathrm{~N}$ can be used by the NMPC. The values of $\gamma, \sigma_{f}, \sigma_{\Phi}$ and $\mathcal{L}_{\tau}$ are the same as in Example 1. In Fig. 5, the evolution of the state and the control variables is shown starting at the same initial condition of Example 1 and 2. The matched disturbance term is shown in Fig. 5 as well, while the unmatched one has the same realization of the unmatched disturbance reported in Fig. 3. Even though now a much larger disturbance is considered (from $\gamma=3.4 \cdot 10^{-3}$ to $\gamma=1$ ), the state trajectory is not distinguishable from the one reported in Fig. 3. Fig. 4 shows a numerical evaluation of the region of attraction using the combined strategy, which coincides with that evaluated for the Example 1. The size of the region is not reduced because the available control amplitude is larger than the one which the NMPC controller needs to find a feasible solution to the FHOCP in the same region of attraction. If the total maximum control amplitude had been smaller, then handling a matched disturbance of non-negligible magnitude would have caused a reduction of the region of attraction (however, remember that using only the NMPC with the full disturbance Assumption 4 is not satisfied, so that a region of attraction does not exist).

\section{CONCLUSION}

In this paper, a hierarchical Model Predictive Control scheme with Integral Sliding Mode for continuous-time nonlinear systems is proposed and analyzed. The contribution of this work can be summarized in the following three points. First, an ISM control strategy is designed in order to reduce the conservativeness of the robust open-loop NMPC strategy. Second, a robust NMPC control strategy with tightened constraints, which generates a piecewise-constant control law, is proposed for continuous-time systems. Third, the concept of regional ISpS property in continuous-time is introduced, and it is proved that the existence of a suitably defined Lyapunov function can guarantee
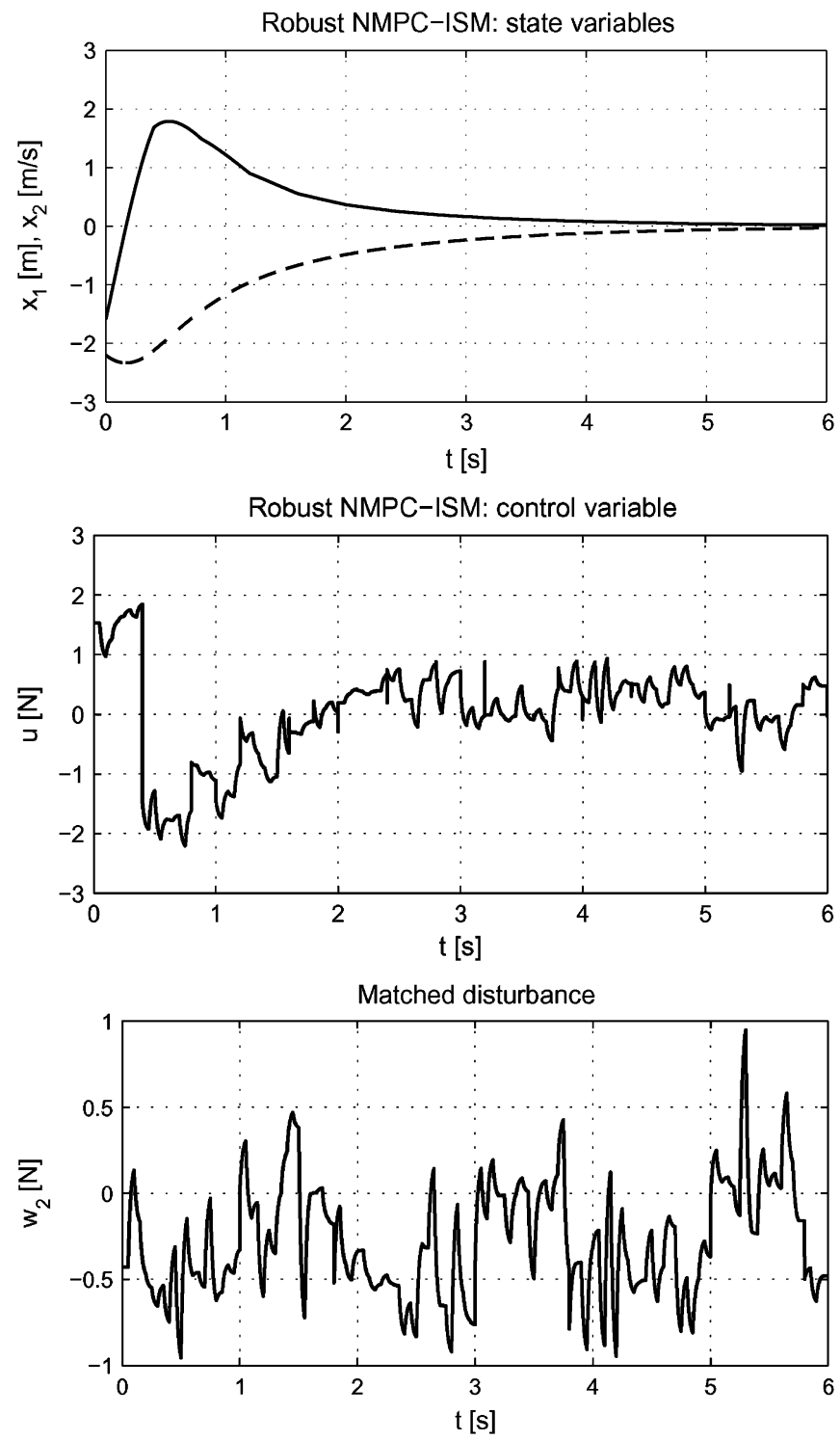

Fig. 5. Time evolution of the state variables ( $x_{1}$ : dashed line, $x_{2}$ : solid line), of the control variable and of the matched disturbance for the NMPC-ISM controller.

the regional ISpS of a system. This concept is then exploited to analyze, under suitable assumptions, the stability properties of the proposed control scheme. Finally, simulation examples are given, showing the advantages of the proposed hierarchical scheme.

\section{APPENDIX A \\ RESUlt ON TightenED SETS}

The following lemma is useful to prove the properties of the proposed NMPC control law.

Lemma 5: Let $x \in \mathcal{X}_{(k+1) T+\tau}, y \in \mathbb{R}^{n}$ such that $|y-x| \leq$ $\gamma T \mathcal{L}_{\tau} \mathcal{L}_{T}$, then $y \in \mathcal{X}_{k T+\tau}$.

Proof: Let $\alpha_{k T+\tau} \in \mathcal{B}_{k T+\tau}$, and $z=y-x+\alpha_{k T+\tau}$. Then, one has that $|z| \leq|y-x|+\left|\alpha_{k T+\tau}\right| \leq \gamma T \mathcal{L}_{\tau} \mathcal{L}_{T}^{k}+\gamma \tau+$ $\gamma T \mathcal{L}_{\tau}\left(\left(\mathcal{L}_{T}^{k}-1\right) /\left(\mathcal{L}_{T}-1\right)\right)=\gamma\left(\tau+T \mathcal{L}_{\tau}\left(\left(\mathcal{L}_{T}^{k}-1\right) /\left(\mathcal{L}_{T}-1\right)\right)\right)$, and so $z \in \mathcal{B}_{(k+1) T+\tau}$. Since $x \in \mathcal{X}_{(k+1) T+\tau}, \forall \alpha_{k T+\tau} \in$ $\mathcal{B}_{k T+\tau}$, it is verified that $y+\alpha_{k T+\tau}=x+z \in \mathcal{X}$. Then $y \in \mathcal{X}_{k T+\tau}$. 


\section{APPENDIX B}

\section{CONTINUOUS-TIME REGIONAL ISPS}

In this subsection the ISS framework for continuous-time autonomous nonlinear systems is presented and Lyapunov-like sufficient conditions are provided. This framework is used to prove the stability properties of the proposed NMPC control scheme. The piecewise-constant control law introduced in Section IV-B will make the closed-loop system time-varying: it is then necessary to deal with time-varying systems.

Consider a time-varying continuous-time autonomous nonlinear dynamic system described by

$$
\dot{x}(t)=F(t, x(t), w(t)), \quad t \geq 0
$$

where $F: \mathbb{R}_{\geq 0} \times \mathbb{R}^{n} \times \mathbb{R}^{p} \rightarrow \mathbb{R}^{n}$ is nonlinear and possibly discontinuous, $x(t) \in \mathbb{R}^{n}$ is the state, $w(t) \in \mathbb{R}^{p}$ is an uncertain term. The solution of system (22) with initial state $x\left(t_{0}\right)=\bar{x}$ and the sequence of the uncertain terms $\mathbf{w}$ is denoted by $\varphi_{F}\left(t, \bar{x}, t_{0}, \mathbf{w}\right)$. This system is supposed to fulfill the following assumption.

\section{Assumption 5:}

1) System (22) is forward complete.

2) The uncertainty $w$ is such that

$$
w \in \mathcal{W}
$$

where $\mathcal{W} \subset \mathbb{R}^{p}$ is a compact set containing the origin with $\mathcal{W}^{\text {sup }}$ known.

Definition 2 (RPI Set): A set $\Xi \subseteq \mathbb{R}^{n}$ is a robust positively invariant (RPI) set for system (22) if $\varphi_{F}\left(t, \bar{x}, t_{0}, \mathbf{w}\right) \in \Xi$, for all $\bar{x} \in \Xi$, all $\mathbf{w} \in \mathcal{M}_{\mathcal{W}}$, all $t \geq t_{0}$, and all $t_{0}>0$.

A regional version of ISpS [40] is now defined.

Definition 3: [Regional ISpS in $\Xi$ ] Given a compact set $\Xi \subseteq$ $\mathbb{R}^{n}$ including the origin as an interior point, system (22) with $\mathbf{w} \in \mathcal{M}_{\mathcal{W}}$ is said to be ISpS (Input-to-State practical Stable) in $\Xi$ with respect to $w$, if $\Xi$ is a RPI set for (22) and if there exist a $\mathcal{K} \mathcal{L}$-function $\beta(\cdot, \cdot)$, a $\mathcal{K}$-function $\tilde{\gamma}(\cdot)$ and a constant $c \geq 0$ such that

$$
\left|\varphi_{F}\left(t, \bar{x}, t_{0}, \mathbf{w}\right)\right| \leq \beta\left(|\bar{x}|, t-t_{0}\right)+\tilde{\gamma}(\|\mathbf{w}\|)+c
$$

for all $\bar{x} \in \Xi$ and $t \geq t_{0}$. Whenever $c=0$, system (22) is said to be ISS (Input-to-State Stable) in $\Xi$ with respect to $w$.

Regional ISpS will be now associated to the existence of a suitable Lyapunov function (in general, a priori, non-continuous) with respect to $w$.

Definition 4 (ISpS-Lyapunov Function in $\Xi$ ): A function $V$ : $\mathbb{R}_{\geq 0} \times \mathbb{R}^{n} \rightarrow \mathbb{R}_{\geq 0}$ is called an ISpS-Lyapunov function in $\Xi$ for system (22) with respect to $w$ if

1) $\Xi$ is a compact RPI set including the origin as an interior point.

2) there exist a pair of suitable $\mathcal{K}_{\infty}$-functions $\alpha_{1}, \alpha_{2}$ and a constant $c_{1} \geq 0$ such that

$$
\begin{aligned}
& V(t, x(t)) \geq \alpha_{1}(|x(t)|), \quad \forall x(t) \in \Xi \\
& V(t, x(t)) \leq \alpha_{2}(|x(t)|)+c_{1}, \quad \forall x(t) \in \Xi .
\end{aligned}
$$

3) there exist a suitable $\mathcal{K}_{\infty}$-function $\alpha_{3}$, a $\mathcal{K}$-function $\sigma$ and a constant $c_{2} \geq 0$ such that

$$
\begin{aligned}
\dot{V}(t, x(t)) & =\frac{\partial V}{\partial t}(t, x(t))+\frac{\partial V}{\partial x}(t, x(t)) F(t, x(t), w(t)) \\
& \leq-\alpha_{3}(|x(t)|)+\sigma(|w(t)|)+c_{2}
\end{aligned}
$$

for all $x(t) \in \Xi$, all $w(t) \in \mathcal{W}$, and for almost all $t$. For the values $\bar{t}_{i}, i \geq 0$, for which (25) does not hold, the following condition holds:

$V\left(\bar{t}_{i}^{+}, x\left(\bar{t}_{i}^{+}\right)\right) \leq V\left(\bar{t}_{i}^{-}, x\left(\bar{t}_{i}^{-}\right)\right), \quad \forall i \geq 0$.

4) there exist suitable $\mathcal{K}_{\infty}$-functions $\zeta$ and $\varrho$ (with $\varrho$ such that $(i d-\varrho)$ is a $\mathcal{K}_{\infty}$-function) such that, given an uncertain signal $\mathbf{w} \in \mathcal{M}_{\mathcal{W}}$, there exists a nonempty compact set $\Theta_{\mathrm{w}} \subseteq \Xi$ (including the origin as an interior point) defined as follows:

$$
\Theta_{\mathbf{w}} \triangleq\{x: V(t, x) \leq \bar{c}, \forall t \geq 0\}
$$

where $\bar{c} \triangleq b\left(\sigma\left(\mathcal{W}^{\text {sup }}\right)+c_{3}\right), b \triangleq \alpha_{4}^{-1} \circ(i d-\varrho)^{-1}$, with $\alpha_{4} \triangleq \underline{\alpha}_{3} \circ \bar{\alpha}_{2}^{-1}, \underline{\alpha}_{3}(s) \triangleq \min \left(\alpha_{3}(s / 2), \zeta(s / 2)\right)$, $\bar{\alpha}_{2} \triangleq \alpha_{2}(s)+s, c_{3} \triangleq c_{2}+\zeta\left(c_{1}\right)$.

Whenever $c_{1}=c_{2}=0, V(t, x(t))$ is said to be an ISSLyapunov function for system (22) in $\Xi$.

Then, the following sufficient condition for regional ISpS of system (22) can be stated.

Theorem 2: If system (22) admits an ISpS-Lyapunov function in $\Xi$ with respect to $w$, then it is ISpS in $\Xi$ and $\lim _{t \rightarrow \infty}\left|x\left(t, \bar{x}, t_{0}, \mathbf{w}\right)\right|_{\Theta_{\mathrm{w}}}=0$.

Proof: Let $\bar{x} \in \Xi$. The proof will be carried out in three steps.

Step 1) First we show that the set $\Theta_{\mathbf{w}}$ defined in (27) is robust positively invariant for system (22). From the definition of $\bar{\alpha}_{2}(s)$ it follows that $\alpha_{2}(|x(t)|)+c_{1} \leq \bar{\alpha}_{2}\left(|x(t)|+c_{1}\right)$. Therefore $V(t, x(t)) \leq \bar{\alpha}_{2}\left(|x(t)|+c_{1}\right)$ and hence $|x(t)|+c_{1} \geq$ $\bar{\alpha}_{2}^{-1}(V(t, x(t))), \forall x(t) \in \Xi$. Moreover (see [41])

$$
\alpha_{3}(|x(t)|)+\zeta\left(c_{1}\right) \geq \underline{\alpha}_{3}\left(|x(t)|+c_{1}\right) \geq \alpha_{4}(V(t, x(t)))
$$

where $\alpha_{4} \triangleq \underline{\alpha}_{3} \circ \bar{\alpha}_{2}^{-1}$ is a $\mathcal{K}_{\infty}$-function. Then, the following holds:

$$
\begin{aligned}
\dot{V}(t, x(t)) & \leq-\alpha_{4}(V(t, x(t)))+\zeta\left(c_{1}\right)+c_{2}+\sigma(|w(t)|) \\
& =-\alpha_{4}(V(t, x(t)))+\sigma(|w(t)|)+c_{3} \\
& \leq-\alpha_{4}(V(t, x(t)))+\sigma\left(\mathcal{W}^{\text {sup }}\right)+c_{3}
\end{aligned}
$$

for all $x(t) \in \Xi$, all $w(t) \in \mathcal{W}$, and for almost all $\mathrm{t}$, being $c_{3} \triangleq c_{2}+\zeta\left(c_{1}\right)$. Let us now assume that $x\left(t_{0}\right) \in \Theta_{\mathbf{w}}$. Then, if $\Theta_{\mathbf{w}}$ is robust positively invariant, $x\left(t, \bar{x}, t_{0}, \mathbf{w}\right) \in \Theta_{\mathbf{w}}, \forall t \geq t_{0}$. In order to prove this claim, assume that this is not true. Then, there exist some $t>t_{0}$ and $\varepsilon>0$ such that $V(t, x(t))>\bar{c}+\varepsilon$. Let $\tau=\inf \left\{t \geq t_{0}, t \neq \bar{t}_{i}: V(t, x(t)) \geq \bar{c}+\varepsilon\right\}$. Then, it follows that:

$$
V(\tau, x(\tau)) \geq \bar{c}=\alpha_{4}^{-1} \circ(i d-\varrho)^{-1}\left(\sigma\left(\mathcal{W}^{\text {sup }}\right)+c_{3}\right)
$$


that implies

$$
(i d-\varrho) \circ \alpha_{4}(V(\tau, x(\tau))) \geq \sigma\left(\mathcal{W}^{s u p}\right)+c_{3} .
$$

By substituting this in (29) we obtain

$$
\left.\frac{d}{d t} V(t, x(t))\right|_{t=\tau} \leq-\varrho \circ \alpha_{4}(V(\tau, x(\tau)))<0
$$

for all $x(\tau) \in \Xi \backslash \Theta_{\mathbf{w}}$, all $w(\tau) \in \mathcal{W}$. Hence, considering also that, where $\dot{V}(t, x(t))$ does not exist, (26) holds, $V(t, x(t)) \geq$ $V(\tau, x(\tau))$ for some $t \in\left(t_{0}, \tau\right)$. This contradicts the minimality of $\tau$, and hence, as claimed, $\Theta_{\mathbf{w}}$ is robust positively invariant (i.e. $x(t) \in \Theta_{\mathbf{w}}$ for all $t \geq t_{0}$ ).

Step 2) Next, we show that the state, starting from $\Xi \backslash \Theta_{\mathbf{w}}$, tends asymptotically to $\Theta_{\mathbf{w}}$. If $x(t) \in \Xi \backslash \Theta_{\mathbf{w}}$, then $(i d-\varrho) \circ$ $\alpha_{4}(V(t, x(t)))>\sigma\left(\mathcal{W}^{\text {sup }}\right)+c_{3}$. Hence

$$
\begin{aligned}
\dot{V}\left(t, x\left(t, \bar{x}, t_{0}, \mathbf{w}\right)\right) & \leq-\varrho \circ \alpha_{4}\left(V\left(t, x\left(t, \bar{x}, t_{0}, \mathbf{w}\right)\right)\right) \\
& \leq-\varrho \circ \alpha_{4} \circ \alpha_{1}\left(\left|x\left(t, \bar{x}, t_{0}, \mathbf{w}\right)\right|\right)
\end{aligned}
$$

for all $x(t) \in \Xi \backslash \Theta_{\mathbf{w}}$, and for almost all $t$, the last step being obtained using (23). Considering also that, where $\dot{V}(t, x(t))$ does not exist, (26) holds, then, $\forall \varepsilon^{\prime}>0, \exists T_{1}\left(\varepsilon^{\prime}\right) \geq t_{0}$ such that

$$
V\left(T_{1}, x\left(T_{1}, \bar{x}, t_{0}, \mathbf{w}\right)\right) \leq \varepsilon^{\prime}+\bar{c} .
$$

Therefore, starting from $\Xi$, the state will arrive close to $\Theta_{\mathbf{w}}$ in a finite time and to $\Theta_{\mathbf{w}}$ asymptotically. Hence $\lim _{k \rightarrow \infty}\left|x\left(k, \bar{x}, \mathbf{d}_{\mathbf{1}}, \mathbf{d}_{\mathbf{2}}\right)\right|_{\Theta_{\mathbf{w}}}=0$.

Step 3) Finally we show that system (22) is regional ISpS in $\Xi$. Let $t_{1}=\inf \left\{t \geq t_{0}: x(t) \in \Theta_{\mathrm{w}}\right\} \leq \infty$. Then, from the robust invariance of $\Theta_{\mathbf{w}}$, it follows that $V(t, x(t)) \leq \alpha_{4}^{-1} \circ(i d-$ $\varrho)^{-1}\left(\sigma\left(\mathcal{W}^{\text {sup }}\right)+c_{3}\right)$, for all $t \geq t_{1}$. By using (23), this implies that

$$
\left|x\left(t, \bar{x}, t_{0}, \mathbf{w}\right)\right| \leq \alpha_{1}^{-1} \circ \alpha_{4}^{-1} \circ(i d-\varrho)^{-1}\left(\sigma\left(\mathcal{W}^{\text {sup }}\right)+c_{3}\right) .
$$

Noting that, given a $\mathcal{K}_{\infty}$-function $\theta_{1}, \theta_{1}\left(s_{1}+s_{2}\right) \leq \theta_{1}\left(2 s_{1}\right)+$ $\theta_{1}\left(2 s_{2}\right)$ (see [41]) it follows that:

$$
\left|x\left(t, \bar{x}, t_{0}, \mathbf{w}\right)\right| \leq \tilde{\gamma}\left(\mathcal{W}^{\text {sup }}\right)+\tilde{c}, \quad \forall t \geq t_{1}
$$

where $\tilde{\gamma}(s) \triangleq \alpha_{1}^{-1} \circ \alpha_{4}^{-1} \circ(i d-\varrho)^{-1}(2 \sigma(s))$ and $\tilde{c} \triangleq \alpha_{1}^{-1} \circ$ $\alpha_{4}^{-1} \circ(i d-\varrho)^{-1}\left(2 c_{3}\right)$. For $t<t_{1}, x(t) \notin \Theta_{\mathbf{w}}$, that implies

$$
\dot{V}\left(t, x\left(t, \bar{x}, t_{0}, \mathbf{w}\right)\right) \leq-\varrho \circ \alpha_{4}\left(V\left(t, x\left(t, \bar{x}, t_{0}, \mathbf{w}\right)\right)\right)
$$

for almost all $t<t_{1}$.

First of all, this inequality guarantees that $x(t)$ is defined for all $t \geq t_{0}$. Secondly, by the following generalization of the comparison principle [42], there exists some $\mathcal{K} \mathcal{L}$-function $\tilde{\beta}$ which only depends on $\varrho$ and $\alpha_{4}$, such that $V(t, x(t)) \leq$ $\tilde{\beta}\left(V\left(t_{0}, \bar{x}\right), t-t_{0}\right)$ for $t \leq t_{1}$.

Define for any $s>0, \eta(s) \triangleq-\int_{1}^{s}\left(d r / \varrho \circ \alpha_{4}(r)\right)$. This is a strictly decreasing differentiable function on $(0, \infty)$, with $\lim _{s \rightarrow 0^{+}} \eta(s)=+\infty$. Let $0<a \triangleq-\lim _{s \rightarrow+\infty} \eta(s)$. Then, the range of $\eta$, and hence the domain of $\eta^{-1}$, is the open interval $(-a, \infty)$ (we allow the possibility that $a=\infty$ ). For $(s, t) \in$ $\mathbb{R}_{\geq 0} \times \mathbb{R}_{\geq 0}$, define

$$
\tilde{\beta}(s, t) \triangleq \begin{cases}0, & \text { if } s=0 \\ \eta^{-1}(\eta(s)+t), & \text { if } s>0 .\end{cases}
$$

In order to verify that $V(t, x(t)) \leq \tilde{\beta}\left(V\left(t_{0}, \bar{x}\right), t-t_{0}\right)$, we only need to show that

$$
\eta(V(t, x(t))) \geq \eta\left(V\left(t_{0}, x\left(t_{0}\right)\right)\right)+t-t_{0}, \quad t_{0} \leq t \leq t_{1}
$$

that is

$$
\begin{aligned}
- & \int_{1}^{V(t, x(t))} \frac{d r}{\varrho \circ \alpha_{4}(r)} \\
& \geq-\int_{1}^{V\left(t_{0}, x\left(t_{0}\right)\right)} \frac{d r}{\varrho \circ \alpha_{4}(r)}+t-t_{0}, \quad t_{0} \leq t \leq t_{1}
\end{aligned}
$$

which is equivalent to

$$
\int_{V(t, x(t))}^{V\left(t_{0}, x\left(t_{0}\right)\right)} \frac{d r}{\varrho \circ \alpha_{4}(r)} \geq t-t_{0}, \quad t_{0} \leq t \leq t_{1} .
$$

By the following change of variables $r=V(\tau, x(\tau))$ and taking into account (26), denoting $z=V\left(\tau, x\left(\tau, \bar{x}, t_{0}, \mathbf{w}\right)\right)$ one sees that the previous inequality is equivalent to

$$
\begin{aligned}
& \int_{t_{0}}^{\bar{t}_{1}^{-}} \frac{\dot{V}(\tau, x(\tau))}{\varrho \circ \alpha_{4}(z)} d \tau+\epsilon_{\bar{t}_{1}}+\int_{\bar{t}_{1}^{+}}^{\bar{t}_{2}^{-}} \frac{\dot{V}(\tau, x(\tau))}{\varrho \circ \alpha_{4}(z)} d \tau+\epsilon_{\bar{t}_{2}}+\ldots \\
& \quad+\int_{\bar{t}_{N}^{+}}^{t_{1}} \frac{\dot{V}(\tau, x(\tau))}{\varrho \circ \alpha_{4}(z)} d \tau \\
& \leq-\int_{t_{0}}^{\bar{t}_{1}^{-}} d \tau-\int_{\bar{t}_{1}^{+}}^{\bar{t}_{2}^{-}} d \tau-\ldots-\int_{\bar{t}_{N}^{+}}^{t} d \tau=t_{0}-t, \quad t_{0} \leq t \leq t_{1}
\end{aligned}
$$

where $\epsilon_{\bar{t}_{i}}$ are due to the jump discontinuities of $V(x(t), t)$ and, for (26), are always negative. By considering (32), the previous inequality is always satisfied. It only remains to show that $\tilde{\beta}$ is of class $\mathcal{K} \mathcal{L}$. The function $\tilde{\beta}$ is continuous since both $\eta$ and $\eta^{-1}$ are continuous in their domains, and $\lim _{r \rightarrow \infty} \eta^{-1}(r)=0$. It is strictly increasing in $s$ for each fixed $t$ since both $\eta$ and $\eta^{-1}$ are strictly decreasing. Finally $\tilde{\beta}(s, t) \rightarrow 0$ as $t \rightarrow \infty$ by construction. So $\tilde{\beta}$ is a $\mathcal{K} \mathcal{L}$-function.

Hence $V(t, x(t)) \leq \tilde{\beta}\left(V\left(t_{0}, \bar{x}\right), t-t_{0}\right), t_{0} \leq t \leq t_{1}$. Вy using (23) and (24), it yields

$$
\left|x\left(t, \bar{x}, t_{0}, \mathbf{w}\right)\right| \leq \beta\left(|\bar{x}|, t-t_{0}\right)+\hat{c}, \quad \forall t \leq t_{1}
$$

where $\beta(s) \triangleq \alpha_{1}^{-1} \circ \tilde{\beta}\left(2 \alpha_{2}(s), t-t_{0}\right)$ and $\hat{c} \triangleq \alpha_{1}^{-1} \circ \tilde{\beta}\left(2 c_{1}, 0\right)$.

Combining (31) and (33), one concludes that the system is ISpS, i.e.

$$
\left|x\left(t, \bar{x}, t_{0}, \mathbf{w}\right)\right| \leq \beta\left(|\bar{x}|, t-t_{0}\right)+\tilde{\gamma}(\|w\|)+c, \quad \forall t \geq t_{0}
$$

where $c \triangleq \tilde{c}+\hat{c}$. 


\section{APPENDIX C \\ OTHER PROOFS}

Proof of Lemma 1: Using (7), if the nominal system and the real one start at the same point at time $t_{k}$, then after a sampling time interval it yields

$$
\left|\varphi\left(T, x\left(t_{k}\right), \mathbf{u}, \mathbf{0}\right)-\varphi\left(T, x\left(t_{k}\right), \mathbf{u}, \mathbf{w}\right)\right| \leq \gamma T .
$$

After two sample time intervals, one has that

$$
\begin{gathered}
\left|\varphi\left(2 T, x\left(t_{k}\right), \mathbf{u}, \mathbf{0}\right)-\varphi\left(2 T, x\left(t_{k}\right), \mathbf{u}, \mathbf{w}\right)\right| \\
=\left|\varphi\left(T, x_{1}, \mathbf{u}, \mathbf{0}\right)-\varphi\left(T, x_{2}, \mathbf{u}, \mathbf{w}\right)\right| \\
=\mid \varphi\left(T, x_{1}, \mathbf{u}, \mathbf{0}\right)-\varphi\left(T, x_{2}, \mathbf{u}, \mathbf{0}\right) \\
\quad+\varphi\left(T, x_{2}, \mathbf{u}, \mathbf{0}\right)-\varphi\left(T, x_{2}, \mathbf{u}, \mathbf{w}\right) \mid
\end{gathered}
$$

where $x_{1} \triangleq \varphi\left(T, x\left(t_{k}\right), \mathbf{u}, \mathbf{0}\right)$ and $x_{2} \triangleq \varphi\left(T, x\left(t_{k}\right), \mathbf{u}, \mathbf{w}\right)$. Using the triangle inequality together with Assumption 2 , it yields

$$
\begin{aligned}
& \left|\varphi\left(2 T, x\left(t_{k}\right), \mathbf{u}, \mathbf{0}\right)-\varphi\left(2 T, x\left(t_{k}\right), \mathbf{u}, \mathbf{w}\right)\right| \\
& \quad \leq \mathcal{L}_{T}\left|\varphi\left(T, x\left(t_{k}\right), \mathbf{u}, 0\right)-\varphi\left(T, x\left(t_{k}\right), \mathbf{u}, \mathbf{w}\right)\right|+\gamma T \\
& \quad \leq \gamma T\left(\mathcal{L}_{T}+1\right) .
\end{aligned}
$$

Analogously, after $i$ sampling times, the result can be obtained as the sum of a geometric series

$$
\left|\varphi\left(i T, x\left(t_{k}\right), \mathbf{u}, \mathbf{0}\right)-\varphi\left(i T, x\left(t_{k}\right), \mathbf{u}, \mathbf{w}\right)\right| \leq \gamma T \frac{\mathcal{L}_{T}^{i}-1}{\mathcal{L}_{T}-1} .
$$

Finally, in order to generalize it for a generic interval $t \triangleq i T+\tau$, it yields

$$
\begin{aligned}
& \left|\varphi\left(i T+\tau, x\left(t_{k}\right), \mathbf{u}, \mathbf{0}\right)-\varphi\left(i T+\tau, x\left(t_{k}\right), \mathbf{u}, \mathbf{w}\right)\right| \\
& \quad \leq \mathcal{L}_{\tau}\left|\varphi\left(i T, x\left(t_{k}\right), \mathbf{u}, \mathbf{0}\right)-\varphi\left(i T, x\left(t_{k}\right), \mathbf{u}, \mathbf{w}\right)\right|+\gamma \tau \\
& \quad \leq \gamma\left(\tau+T \mathcal{L}_{\tau} \frac{\mathcal{L}_{T}^{i}-1}{\mathcal{L}_{T}-1}\right) .
\end{aligned}
$$

Proof of Lemma 3: To get the feasibility property, one has to prove that

$$
\begin{aligned}
x\left(t_{k}\right) & \in \mathcal{X}^{M P C} \Rightarrow \varphi\left(T, x\left(t_{k}\right), \kappa^{M P C}\left(x\left(t_{k}\right)\right), \mathbf{w}_{\left[t_{k}, t_{k+1}\right)}\right) \\
& \in \mathcal{X}^{M P C} .
\end{aligned}
$$

Letting $x\left(t_{k}\right) \in \mathcal{X}^{M P C}$ and the associated optimal solution $\overline{\mathbf{u}}_{\left[t_{k}, t_{k+N-1} \mid t_{k}\right]}^{o}$ of the FHOCP at time $t_{k}$, a possible (sub-optimal) solution at time $t_{k+1}$ for the FHOCP is

$$
\begin{aligned}
& \overline{\mathbf{u}}_{\left[t_{k+1}, t_{k+N} \mid t_{k+1}\right]}^{S} \overline{\mathbf{u}}_{\left[t_{k+1}, t_{k+N-1} \mid t_{k}\right]}^{o} \\
& \triangleq\left[\begin{array}{c}
\kappa_{f}\left(\varphi\left((N-1) T, x\left(t_{k+1}\right), \mathbf{u}_{\left[t_{k+1}, t_{k+N} \mid t_{k}\right)}^{o}, \mathbf{0}\right)\right)
\end{array}\right]
\end{aligned}
$$

where

$$
x\left(t_{k+1}\right) \triangleq\left(\varphi\left(T, x\left(t_{k}\right), \kappa^{M P C}\left(t_{k}\right), \mathbf{w}_{\left[t_{k}, t_{k+1}\right)}\right)\right)
$$

is the value of the state of the NMPC perturbed closed-loop system at time $t_{k+1}$. To determine the feasibility of such a solution, one must prove the following three steps.

Step 1) it is necessary to show that the state value must lay in $\mathcal{X}_{f}$ at $t_{k+N+1}$, i.e.

$$
\varphi\left(N T, x\left(t_{k+1}\right), \mathbf{u}_{\left[t_{k+1}, t_{k+N+1} \mid t_{k+1}\right)}^{s}, \mathbf{0}\right) \in \mathcal{X}_{f}
$$

where $\mathbf{u}_{\left[t_{k+1}, t_{k+N+1} \mid t_{k+1}\right)}^{s}$ is the signal associated with the control sequence $\overline{\mathbf{u}}_{\left[t_{k+1}, t_{k+N} \mid t_{k+1}\right]}$. In order to prove this, we show that $\varphi\left((N-1) T, x\left(t_{k+1}\right), \mathbf{u}_{\left[t_{k+1}, t_{k+N} \mid t_{k+1}\right)}^{s}, \mathbf{0}\right) \in \Phi$. To this aim, note that Assumption 2 implies

$$
\begin{aligned}
& \mid \varphi\left((N-1) T, x\left(t_{k+1}\right), \mathbf{u}_{\left[t_{k+1}, t_{k+N} \mid t_{k+1}\right)}^{s}, \mathbf{0}\right)- \\
& \quad \varphi\left((N-1) T, x\left(t_{k+1} \mid t_{k}\right), \mathbf{u}_{\left[t_{k+1}, t_{k+N} \mid t_{k+1}\right)}^{s}, \mathbf{0}\right) \mid \leq \gamma T \mathcal{L}_{T}^{N-1}
\end{aligned}
$$

where

$$
x\left(t_{k+1} \mid t_{k}\right) \triangleq \varphi\left(T, x\left(t_{k}\right), \mathbf{u}_{\left[t_{k}, t_{k+1} \mid t_{k}\right)}^{o}, \mathbf{0}\right)
$$

is the value of the state of the NMPC nominal (without uncertainties) closed-loop system at time $t_{k+1}$. Since $\varphi((N-$ 1)T, $\left.x\left(t_{k+1} \mid t_{k}\right), \mathbf{u}_{\left[t_{k+1}, t_{k+N} \mid t_{k+1}\right)}^{s}, \mathbf{0}\right) \in \mathcal{X}_{f}$, by defining

$$
\varphi_{p} \triangleq \varphi\left((N-1) T, x\left(t_{k+1}\right), \mathbf{u}_{\left[t_{k+1}, t_{k+N} \mid t_{k+1}\right)}^{s}, \mathbf{0}\right)
$$

and

$$
\varphi_{n} \triangleq \varphi\left((N-1) T, x\left(t_{k+1} \mid t_{k}\right), \mathbf{u}_{\left[t_{k+1}, t_{k+N} \mid t_{k+1}\right)}^{s}, \mathbf{0}\right)
$$

it yields

$$
V_{f}\left(\varphi_{p}\right)-V_{f}\left(\varphi_{n}\right) \leq\left|V_{f}\left(\varphi_{p}\right)-V_{f}\left(\varphi_{n}\right)\right| \leq L_{f}\left|\varphi_{p}-\varphi_{n}\right|
$$

and then

$$
V_{f}\left(\varphi_{p}\right) \leq V_{f}\left(\varphi_{n}\right)+L_{f} \gamma T \mathcal{L}_{T}^{N-1} \leq \sigma_{f}+L_{f} \gamma T \mathcal{L}_{T}^{N-1} \leq \sigma_{\Phi}
$$

where the last inequality is obtained applying (21). At this point, applying $\kappa_{f}$, according to Assumption 3, one obtains $\varphi\left(N T, x\left(t_{k+1}\right), \mathbf{u}_{\left[t_{k+1}, t_{k+N+1} \mid t_{k+1}\right)}^{s}, \mathbf{0}\right) \in \mathcal{X}_{f}$.

Step 2) the control variable must fulfill $\mathbf{u}_{\left[t_{k+1}, t_{k+N+1} \mid t_{k+1}\right)} \in$ $\overline{\mathcal{U}}$. It follows from the fact that $\mathbf{u}_{\left[t_{k}, t_{k+N} \mid t_{k}\right)}^{o} \in \mathcal{\mathcal { U }}$ by definition, and $\kappa_{f}\left(x\left(t_{k+N}\right)\right) \in \overline{\mathcal{U}}$, since $x\left(t_{k+N}\right) \in \Phi$.

Step 3 ) in order to assure the respect of the state constraints, it must be verified that

$$
\varphi\left((j-1) T+\tau, x\left(t_{k+1}\right), \mathbf{u}_{\left[t_{k+1}, t_{k+j}+\tau \mid t_{k+1}\right)}^{s}, \mathbf{0}\right) \in \mathcal{X}_{(j-1) T+\tau}
$$

for all time instants in the prediction horizon, i.e. $\tau \in(0, T]$ and $j=1,2, \ldots, N$. Considering that $\mid \varphi\left(T, x\left(t_{k+1}\right), \mathbf{u}_{\left[t_{k+1}, t_{k+2} \mid t_{k+1}\right)}^{s}, \mathbf{0}\right)$ $\varphi\left(T, x\left(t_{k+1} \mid t_{k}\right), \mathbf{u}_{\left[t_{k+1}, t_{k+2} \mid t_{k+1}\right)}^{s}, \mathbf{0}\right) \mid \stackrel{\left.t_{k+1}, t_{k+2} \mid t_{k+1}\right)}{\leq} \quad \gamma T$, then by recursion, for all $\tau \in(0, T]$ and for $j=1,2, \ldots, N$, considering Assumption 3 , it yields $\mid \varphi\left((j-1) T+\tau, x\left(t_{k+1}\right), \mathbf{u}_{\left[t_{k+1}, t_{k+j}+\tau \mid t_{k+1}\right)}^{s}, \mathbf{0}\right)-\varphi((j-$ 


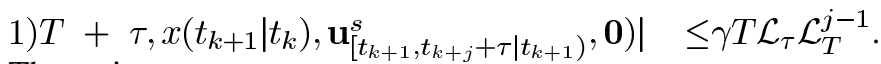
Then, since

$\varphi\left((j-1) T+\tau, x\left(t_{k+1} \mid t_{k}\right), \mathbf{u}_{\left[t_{k+1}, t_{k+j}+\tau \mid t_{k+1}\right)}^{s}, \mathbf{0}\right) \in \mathcal{X}_{j T+\tau}$,

according to Lemma 5 (Appendix A.), it yields

$\varphi\left((j-1) T+\tau, x\left(t_{k+1}\right), \mathbf{u}_{\left[t_{k+1}, t_{k+j}+\tau \mid t_{k+1}\right)}^{s}, \mathbf{0}\right) \in \mathcal{X}_{(j-1) T+\tau}$.

Proof of Lemma 4: In order to prove the regional ISpS of the closed loop system, first note that Assumption 5 is satisfied for system (2). In order to prove the stability properties one has to find a suitable ISpS Lyapunov function, as described in Theorem 2. At any time instant $t \triangleq t_{k}+\tau$, define

$$
x_{c}(t) \triangleq\left[\varphi_{x}\left(\tau, x_{c}\left(t_{k}\right), \mathbf{w}_{\left[t_{k}, t\right)}\right)^{T} \quad \varphi_{u}\left(\tau, x_{x}\left(t_{k}\right), \mathbf{0}\right)^{T}\right]_{(40)}^{T}
$$

as the value of the extended state of the NMPC perturbed closed-loop system at time $t$, where $x_{c}\left(t_{k}\right)$ is the extended state defined in Section III, as well as $\varphi_{x}$ and $\varphi_{u}$. Then, for a fixed value of $N$, introduce the following ISpS Lyapunov function candidate:

$$
\begin{aligned}
V\left(t, x_{c}(t), N\right) \triangleq & \int_{0}^{T-\tau} l\left(\varphi_{x}\left(\zeta, x_{c}(t), \mathbf{0}\right), \varphi_{u}\left(\zeta, x_{c}(t), \mathbf{0}\right)\right) d \zeta \\
& +J\left(\varphi\left(T-\tau, x_{c}(t), \mathbf{0}\right),\right. \\
& \left.\quad \overline{\mathbf{u}}_{\left[t_{k+1}, t_{k+N-1} \mid t_{k}\right]}^{o}, N-1\right) \\
& t \in\left[t_{k}, t_{k+1}\right)
\end{aligned}
$$

which is defined at any time value, and takes into account the remaining part of the stage cost until the end of the prediction horizon. In this way, the length of the integral is varying from $(N-1) T$ at the left neighborhood of each sampling instant $t_{k}$, to $N T$ at each sampling instant. This choice of the Lyapunov function is similar to the one made in [29] for the nominal case.

Now we verify that this function satisfies all the points in Definition 4.

Point 1 is fulfilled, because $\mathcal{X}^{M P C} \supset \mathcal{X}_{f}$ includes the origin as interior point, and is a robust positively invariant set according to Lemma 3.

Point 2 requires to find two $\mathcal{K}_{\infty}$-functions $\alpha_{1}(|x|)$ and $\alpha_{2}(|x|)$ which are a lower and an upper bound, respectively, for the ISpS Lyapunov function candidate. As for the lower bound, one can see that, at $t \in\left[t_{k}, t_{k+1}\right)$

$$
\begin{aligned}
& V\left(t, x_{c}(t), N\right) \\
& \geq \int_{0}^{T-\tau}\left|\varphi_{x}\left(\zeta, x_{c}(t), \mathbf{0}\right)\right|_{Q}^{2} d \zeta \\
& \quad+\int_{0}^{(N-1) T}\left|\varphi\left(\zeta, x\left(t_{k+1}\right), \mathbf{u}_{\left[t_{k+1}, t_{k+1}+\zeta \mid t_{k}\right)}^{o}, \mathbf{0}\right)\right|_{Q}^{2} d \zeta
\end{aligned}
$$

$$
\begin{aligned}
& \geq \lambda_{\min }(Q) \\
& \quad \times\left[\int_{0}^{T-\tau}\left|\varphi_{x}\left(\zeta, x_{c}(t), \mathbf{0}\right)\right|^{2} d \zeta\right. \\
& \left.\quad+\int_{0}^{(N-1) T}\left|\varphi\left(\zeta, x\left(t_{k+1}\right), \mathbf{u}_{\left[t_{k+1}, t_{k+1}+\zeta \mid t_{k}\right)}^{o}, \mathbf{0}\right)\right|^{2} d \zeta\right] .
\end{aligned}
$$

Being $a_{d} \triangleq \max _{x \in \mathcal{X}, u \in \overline{\mathcal{U}}, w \in \mathcal{W}}|h(x)+B u+w|$ one can see that a lower bound on the integral of the state evolution can be obtained if the state moves towards the origin with a "velocity" equal to $a_{d}$ and, if the origin is reached, it remains there. Taking into account the evolution of the system with this maximum derivative, the value of the integral is smaller if calculated on a smaller interval, so, the minimum amount of this interval, i.e. $T^{*} \triangleq(N-1) T$ is considered. This means that we can write

$$
\begin{aligned}
& \lambda_{\min }(Q) {\left[\int_{0}^{T-\tau}\left|\varphi_{x}\left(\zeta, x_{c}(t), \mathbf{0}\right)\right|^{2} d \zeta\right.} \\
&\left.+\int_{0}^{(N-1) T}\left|\varphi\left(\zeta, x\left(t_{k+1}\right), \mathbf{u}_{\left[t_{k+1}, t_{k+1}+\zeta \mid t_{k}\right.}^{o}, \mathbf{0}\right)\right|^{2} d \zeta\right] \\
& \geq \lambda_{\min }(Q) \int_{0}^{t^{*}}\left(|x(t)|-a_{d} \zeta\right)^{2} d \zeta
\end{aligned}
$$

where

$$
t^{*} \triangleq\left\{\begin{array}{lll}
\frac{|x(t)|}{a_{d}}, & |x(t)| \leq a_{d} T^{*} & \text { (case } a) \\
T^{*}, & |x(t)|>a_{d} T^{*} & \text { (case } b)
\end{array}\right.
$$

In case $a$, where the state reaches the origin before the end of the interval $\left[t, t+T^{*}\right)$, solving the integral, one has

$$
\lambda_{\min }(Q) \int_{t_{k}}^{t^{*}}\left(|x(t)|-a_{d} \zeta\right)^{2} d \zeta=\lambda_{\min }(Q) \frac{|x(t)|^{3}}{3 a_{d}}
$$

In case $b$, where the state does not reach the origin in the considered interval, solving the integral it yields

$$
\begin{aligned}
\lambda_{\min }(Q) & \int_{t_{k}}^{t^{*}}\left(|x(t)|-a_{d} \zeta\right)^{2} d \zeta \\
& =\lambda_{\min }(Q)\left(|x(t)|^{2} T^{*}+a_{d}^{2} \frac{T^{* 3}}{3}-a_{d}\left|x\left(t_{k}\right)\right| T^{* 2}\right) .
\end{aligned}
$$

After noting that the two functions coincide at $|x|=a_{d} T^{*}$, that they are strictly increasing in their argument, that the function in case $a$ is equal to zero for $|x|=0$, and that the 
function in case $b$ tends to infinity for $|x| \rightarrow \infty$, it is possible to state that

$$
\alpha_{1}(|x|) \triangleq\left\{\begin{array}{rr}
\lambda_{\min }(Q)|x|^{3} / 3 a_{d}, & |x| \leq a_{d} T^{*} \\
\lambda_{\min }(Q)\left(|x|^{2} T^{*}+a_{d}^{2} \frac{T^{* 3}}{3}\right. & \\
\left.-a_{d}|x| T^{* 2}\right), & |x|>a_{d} T^{*}
\end{array}\right.
$$

is a $\mathcal{K}_{\infty}$-function, such that $V\left(t, x_{c}(t), N\right) \geq \alpha_{1}(|x|)$.

Now, a procedure to find the upper-bound $\alpha_{2}(|x|)$ is introduced. To begin with, define

$$
\begin{aligned}
& \overline{\mathbf{u}}_{\left[t_{k}, t_{k+\tilde{N}-1} \mid t_{k}\right]} \\
& \triangleq \begin{cases}{\left[\begin{array}{cl}
\overline{\mathbf{u}}_{\left[t_{k}, t_{k+\tilde{N}-2} \mid t_{k}\right]}^{o} \\
\kappa_{f}\left(\varphi\left(t_{k+\tilde{N}-1}, x(t), \mathbf{u}_{\left[t, t_{k+\tilde{N}-1} \mid t_{k}\right)}^{o}, \mathbf{0}\right)\right)
\end{array}\right],} & \tilde{N} \geq 2 \\
\kappa_{f}\left(x\left(t_{k}\right)\right), & \tilde{N}=1\end{cases}
\end{aligned}
$$

where $x(t) \triangleq \varphi_{x}\left(\tau, x\left(t_{k}\right), \mathbf{u}_{\left[t_{k}, t \mid t_{k}\right)}, \mathbf{w}_{\left[t_{k}, t\right)}^{o}\right)$. Define also

$$
\begin{aligned}
x\left(t_{k+N} \mid t\right) & \triangleq \varphi\left(N T-\tau, x(t), \mathbf{u}_{\left[t_{k}, t_{k+N} \mid t_{k}\right)}^{o}, \mathbf{0}\right) \\
x_{c}\left(t_{k+N} \mid t\right) & \triangleq\left[x\left(t_{k+N} \mid t\right)^{T} \quad \kappa_{f}\left(x\left(t_{k+N} \mid t\right)\right)^{T}\right]^{T} .
\end{aligned}
$$

The following cost function with horizon $N+1$ is defined, for $t \in\left[t_{k}, t_{k+1}\right)$

$$
\begin{aligned}
& J\left(x(t), \overline{\mathbf{u}}_{\left[t_{k}, t_{k+N} \mid t_{k}\right]}^{e}, N+1\right) \\
& \triangleq V\left(t, x_{c}(t), N\right) \\
& \quad+\int_{t_{k+N}}^{t_{k+N+1}^{+1}} l\left(\varphi\left(\zeta, x\left(t_{k+N} \mid t\right), \mathbf{u}_{\left[t_{k+N}, t_{k+N+\zeta} \mid t_{k}\right)}^{e}, \mathbf{0}\right)\right. \\
& \quad+V_{f}\left(\varphi\left(T, x\left(t_{k+N} \mid t\right), \mathbf{u}_{\left[t_{k+N}, t_{k+N+1} \mid t_{k}\right)}^{e}, \mathbf{0}\right)\right) \\
& \quad-V_{f}\left(x\left(t_{k+N} \mid t\right)\right) .
\end{aligned}
$$

Relying on Point 6 in Assumption 3, it yields

$$
J\left(x(t), \overline{\mathbf{u}}_{\left[t_{k}, t_{k+N}\right]}^{e}, N+1\right) \leq V\left(t, x_{c}(t), N\right) .
$$

By exploiting the optimality of the FHOCP solution, it is possible to state that $V\left(t, x_{c}(t), N+1\right) \leq$ $J\left(x(t), \overline{\mathbf{u}}_{\left[t_{k}, t_{k+N} \mid t_{k}\right]}^{e}, N+1\right) \leq V\left(t, x_{c}(t), N\right) \leq$ $J\left(x(t), \overline{\mathbf{u}}_{\left[t_{k}, t_{k+N-1} \mid t_{k}\right]}^{e}, N\right) \leq \ldots \leq V\left(t, x_{c}(t), 1\right) \leq$ $J\left(x(t), \overline{\mathbf{u}}_{\left[t_{k}, t_{k} \mid t_{k}\right]}^{\left[l_{k}, 1\right)}=J\left(x(t), \kappa_{f}\left(x\left(t_{k}\right)\right), 1\right)\right.$. This inequality holds only for $x\left(t_{k}\right) \in \Phi$, that is the region where a feasible solution for the FHOCP with prediction horizon $N=1$ surely exists.

As a preliminary result, note that, taking into account (6), (18), (19), given $\xi \in[0, T-\tau)$, one can note that $\varphi(\xi, 0,0,0)=$ 0 , and then

$$
\begin{aligned}
\mid \varphi & \left(\xi, x(t), \mathbf{u}_{\left[t, t+\xi \mid t_{k}\right)}^{e}, \mathbf{0}\right) \mid \\
\leq & \left|\varphi\left(\xi, x(t), \mathbf{u}_{\left[t, t+\xi \mid t_{k}\right)}^{e}, \mathbf{0}\right)-\varphi\left(\xi, 0, \mathbf{u}_{\left[t, t+\xi \mid t_{k}\right)}^{e}, \mathbf{0}\right)\right| \\
\quad & +\left|\varphi\left(\xi, 0, \mathbf{u}_{\left[t, t+\xi \mid t_{k}\right)}^{e}, \mathbf{0}\right)-\varphi(\xi, 0,0, \mathbf{0})\right| \\
\leq & \mathcal{L}_{\xi}|x(t)|+\mathcal{L}_{u_{\xi}}\left|\kappa_{f}\left(x\left(t_{k}\right)\right)\right|
\end{aligned}
$$

$$
\begin{aligned}
& =\mathcal{L}_{\xi}|x(t)|+\mathcal{L}_{u_{\xi}}\left|\kappa_{f}\left(x\left(t_{k}\right)\right)-\kappa_{f}(0)\right| \\
& \leq \mathcal{L}_{\xi}|x(t)|+\mathcal{L}_{u_{\xi}} L_{\kappa}\left|x\left(t_{k}\right)\right| .
\end{aligned}
$$

Using (15), (16), (18), (20) and (45), it yields

$$
\begin{aligned}
J\left(x(t), \kappa_{f}\left(x\left(t_{k}\right)\right), 1\right) & \int_{0}^{T-\tau}\left[l\left(\varphi\left(\zeta, x(t), \mathbf{u}_{\left[t, t+\zeta \mid t_{k}\right)}^{e}, \mathbf{0}\right), \kappa_{f}\left(x\left(t_{k}\right)\right)\right)\right. \\
= & -l\left(\varphi\left(\zeta, x(t), \mathbf{u}_{\left[t, t+\zeta \mid t_{k}\right)}^{e}, \mathbf{0}\right), 0\right) \\
& \left.\quad+l\left(\varphi\left(\zeta, x(t), \mathbf{u}_{\left[t, t+\zeta \mid t_{k}\right)}^{e}, \mathbf{0}\right), 0\right)+l(0,0)\right] d \zeta \\
& +V_{f}\left(\varphi\left(T-\tau, x(t), \mathbf{u}_{\left[t, t_{k+1} \mid t_{k}\right)}^{e}, \mathbf{0}\right)\right)-V_{f}(0) \\
\leq & \int_{0}^{T-\tau}\left(L_{l u}\left|\kappa_{f}\left(x\left(t_{k}\right)\right)-\kappa_{f}(0)\right|\right. \\
& \left.\quad+L_{l}\left|\varphi\left(\zeta, x(t), \mathbf{u}_{\left[t, t+\zeta \mid t_{k}\right.}^{e}, \mathbf{0}\right)\right|\right) d \zeta \\
& +L_{f}\left|\varphi\left(T-\tau, x(t), \mathbf{u}_{\left[t, t_{k+1} \mid t_{k}\right)}^{e}, \mathbf{0}\right)\right| \\
\leq & \int_{0}^{T-\tau}\left(L_{l u} L_{\kappa}\left|x\left(t_{k}\right)\right|+L_{l} \mathcal{L}_{\zeta}|x(t)|\right. \\
& \left.\quad+L_{l} \mathcal{L}_{u_{\zeta}} L_{\kappa}\left|x\left(t_{k}\right)\right|\right) d \zeta \\
& +L_{f} \mathcal{L}_{T-\tau}|x(t)|+L_{f} \mathcal{L}_{u_{T-\tau}} L_{\kappa}\left|x\left(t_{k}\right)\right| \\
\leq & T L_{l u} L_{\kappa}\left|x\left(t_{k}\right)\right|+T L_{l} \mathcal{L}_{T}|x(t)|+T L_{l} \mathcal{L}_{u_{T}} L_{\kappa}\left|x\left(t_{k}\right)\right| \\
& +L_{f} \mathcal{L}_{T}|x(t)|+L_{f} \mathcal{L}_{u_{T}} L_{\kappa}\left|x\left(t_{k}\right)\right| \\
\leq & L_{a}\left|x\left(t_{k}\right)\right|+L_{b}|x(t)|
\end{aligned}
$$

with $L_{a} \triangleq T L_{l u} L_{\kappa}+T L_{l} \mathcal{L}_{u_{T}} L_{\kappa}+L_{f} \mathcal{L}_{u_{T}} L_{\kappa}$, and $L_{b} \triangleq$ $T L_{l} \mathcal{L}_{T}+L_{f} \mathcal{L}_{T}$. Since $\Phi$ a compact set, then the value of $L_{a}\left|x\left(t_{k}\right)\right|$ can be bounded as $L_{a}\left|x\left(t_{k}\right)\right| \leq c_{1}, c_{1}>0$. Then, defining $\tilde{\alpha}_{2}(|x|) \triangleq L_{b}|x|$, it yields

$$
V\left(t, x_{c}(t), N\right) \leq \tilde{\alpha}_{2}(|x|)+c_{1}, \quad \forall x \in \Phi .
$$

In order to obtain the upper bound of $V\left(t, x_{c}(t), N\right)$ in the region $\mathcal{X}^{M P C}$, we recall the arguments stated in [41]. The compactness of $\mathcal{X}$ and $\overline{\mathcal{U}}$ implies that the predicted evolution of the system and the feasible control action are bounded. This fact and Assumption 3 guarantee that the optimal cost is upper bounded, that is, there exists a finite real number $\bar{J}$ such that $J\left(x, \overline{\mathbf{u}}_{\left[t_{k}, t_{k+N-1} \mid t_{k}\right]}^{\mathbf{O}}, N\right) \leq \bar{J}, \forall x \in \mathcal{X}_{M P C}$. Let $B_{r} \subset \mathbb{R}^{n}$, $B_{r}=\left\{x \in \mathbb{R}^{n}:|x| \leq r\right\}$, be a ball such that $B_{r} \subset \mathcal{X}_{f}$. Note that this ball exists since the origin is in the interior of $\mathcal{X}_{f}$. Let $\varepsilon$ be a positive constant $\varepsilon=\max \left(1, \bar{J} / \tilde{\alpha}_{2}(r)\right)$. Then, defining $\alpha_{2}(|x|) \triangleq \varepsilon \cdot \tilde{\alpha}_{2}(|x|)$, one has

$$
V\left(t, x_{c}(t), N\right) \leq \alpha_{2}(|x|)+c_{1}, \quad \forall x \in \mathcal{X}^{M P C} .
$$

In fact, if $x \notin \Phi$, then $x \notin B_{r}$ and hence $\tilde{\alpha_{2}}(|x|)>\tilde{\alpha_{2}}(r)$. Then

$$
\begin{aligned}
V\left(t, x_{c}(t), N\right) & \leq \bar{J} \leq \bar{J} \cdot \frac{\tilde{\alpha_{2}}(|x|)}{\tilde{\alpha_{2}}(r)} \\
& \leq \alpha_{2}(|x|)+c_{1}, \quad \forall x \in \mathcal{X}^{M P C} .
\end{aligned}
$$


Point 3 requires to express the derivative of the ISpS Lyapunov function candidate. In the following the right derivative is analyzed (the calculation of the left derivative is specular). Such a derivative is defined as

$$
\begin{aligned}
& \dot{V}\left(t, x_{c}(t), N\right) \\
& \quad \triangleq \lim _{h \rightarrow 0^{+}} \frac{V\left(t+h, x_{c}(t+h), N\right)-V\left(t, x_{c}(t), N\right)}{h} .
\end{aligned}
$$

Note that $t=t_{k}+\tau$. Then, by using the definition of $x_{c}(t)$ in (40), in addition to

$$
x_{c}(t+h) \triangleq\left[\begin{array}{c}
\varphi_{x}\left(\tau+h, x_{c}\left(t_{k}\right), \mathbf{w}_{\left[t_{k}, t+h\right)}\right) \\
\varphi_{u}\left(\tau+h, x_{c}\left(t_{k}\right), \mathbf{0}\right)
\end{array}\right]
$$

the term on the numerator of (46), provided that $[t, t+h) \subseteq$ $\left[t_{k}, t_{k+1}\right)$, can be bounded as follows:

$$
\begin{gathered}
V\left(t+h, x_{c}(t+h), N\right)-V\left(t, x_{c}(t), N\right) \\
=-\int_{0}^{h} l\left(\varphi_{x}\left(\zeta, x_{c}(t), \mathbf{0}\right), \varphi_{u}\left(\zeta, x_{c}(t), \mathbf{0}\right)\right) d \zeta \\
+\int_{0}^{T-(\tau+h)}\left[l\left(\varphi_{x}\left(\zeta, x_{c}(t+h), \mathbf{0}\right), \varphi_{u}\left(\zeta, x_{c}(t+h), \mathbf{0}\right)\right)\right. \\
-l\left(\varphi_{x}\left(\zeta, x_{c}(t+h \mid t), \mathbf{0}\right),\right. \\
\left.\left.\varphi_{u}\left(\zeta, x_{c}(t+h \mid t), \mathbf{0}\right)\right)\right] d \zeta \\
+\int_{0}^{(N-1) T}\left[l \left(\varphi\left(\zeta, x\left(t_{k+1} \mid t+h\right), \mathbf{u}_{\left[t_{k+1}, t_{k+1}+\zeta \mid t_{k}\right)}^{o}, \mathbf{0}\right),\right.\right. \\
\left.\mathbf{u}_{\left[t_{k+1}, t_{k+N} \mid t_{k}\right)}^{o}\left(t_{k+1}+\zeta\right)\right) \\
-l\left(\varphi\left(\zeta, x\left(t_{k+1} \mid t\right), \mathbf{u}_{\left[t_{k+1}, t_{k+1}+\zeta \mid t_{k}\right)}^{o}, \mathbf{0}\right),\right. \\
\left.\left.\mathbf{u}_{\left[t_{k+1}, t_{k+N} \mid t_{k}\right)}^{o}\left(t_{k+1}+\zeta\right)\right)\right] d \zeta \\
+V_{f}\left(\varphi\left((N-1) T, x\left(t_{k+1} \mid t+h\right), \mathbf{u}_{\left[t_{k+1}, t_{k+N} \mid t_{k}\right)}^{o}, \mathbf{0}\right)\right) \\
-V_{f}\left(\varphi\left((N-1) T, x\left(t_{k+1} \mid t\right), \mathbf{u}_{\left[t_{k+1}, t_{k+N} \mid t_{k}\right)}^{o}, \mathbf{0}\right)\right) \cdot(47)
\end{gathered}
$$

Taking into account that the value of the integrand in the second line of (47) is defined for any time instant in $[t, t+h]$ and is also bounded, define $\delta$ as its mean value in $[t, t+h]$. By virtue of (6), (7), (15), (20), after some calculation it yields

$$
\begin{gathered}
V\left(t+h, x_{c}(t+h), N\right)-V\left(t, x_{c}(t), N\right) \\
\leq h\left[\begin{array}{c}
t_{k+N} \\
-\delta
\end{array} \int_{t+h}^{t_{k+h}} \mathcal{L}_{\zeta-(t+h)}^{*} d \zeta\right. \\
\left.\quad+L_{f} \gamma \mathcal{L}_{t_{k+1}-(t+h)} \mathcal{L}_{T}^{N-1}\right]
\end{gathered}
$$

where

$$
\mathcal{L}_{\zeta-(t+h)}^{*} \triangleq \begin{cases}\mathcal{L}_{\zeta-(t+h)}, & \zeta \in\left[t+h, t_{k+1}\right] \\ \mathcal{L}_{\zeta-t} \mathcal{L}_{T}^{\left\lfloor\frac{t-t_{k+1}}{T}\right\rfloor} \mathcal{L}_{\zeta-\left\lfloor\frac{t-t_{k+1}}{T}\right\rfloor,} \quad \zeta \in\left[t_{k+1}, t_{k+N}\right] .\end{cases}
$$

Substituting this upper-bound into (46), solving the limit (taking into account that if $h \rightarrow 0$, then $\delta$ coincides with the value of the integrand in the second line of (47) at time $t$ ), one obtains

$\dot{V}\left(t, x_{c}(t), N\right) \leq-l\left(x(t), \kappa^{M P C}\left(x\left(t_{k}\right)\right)\right)$

$$
+\gamma\left[L_{l} \int_{t}^{t_{k+N}^{N}} \mathcal{L}_{\zeta-t}^{*} d \tau+L_{f} \mathcal{L}_{t_{k+1}-t} \mathcal{L}_{T}^{N-1}\right] .
$$

It is worth noting that the value of $l$ in this case can be calculated considering only the current values of the state and the control variable, independently from the past trajectory of the system. Moreover, this term is defined so that

$$
l(x(t), u(t)) \geq l(x(t), 0)=|x(t)|_{Q}^{2} \geq \lambda_{\min }(Q)|x|^{2} .
$$

As for the term in the second line of (48), a time-invariant upper bound can be obtained substituting $t_{k}$ to $t$. So, after defining

$$
\alpha_{3}(|x|) \triangleq \lambda_{\min }(Q)|x|^{2}
$$

and

$$
\sigma(|w|) \triangleq \gamma\left[L_{l} \int_{t_{k}}^{t_{k+N}} \mathcal{L}_{\tau-t_{k}}^{*} d \tau+L_{f} \mathcal{L}_{T}^{N}\right]
$$

where $\alpha_{3}(|x|)$ is a $\mathcal{K}_{\infty}$-function and $\sigma(|w|)$ is a $\mathcal{K}$-function, one has

$$
\dot{V}\left(t, x_{c}(t), N\right) \leq-\alpha_{3}(|x|)+\sigma(|w|) .
$$

Note that the derivative of the Lyapunov function is defined only almost everywhere, because at the sampling time instants $t_{k}$ a jump discontinuity of $V\left(t, x_{c}(t), N\right)$ can occur. Then, it is necessary to show that $V\left(t_{k}^{+}, x_{c}\left(t_{k}^{+}\right), N\right) \leq V\left(t_{k}^{-}, x_{c}\left(t_{k}^{-}\right), N\right), \forall k$. Considering of having to solve the FHOCP at time $t_{k+1}$, a feasible (sub-optimal) solution can be obtained from the control sequence defined in (35). The corresponding value of the ISpS Lyapunov function candidate at $t_{k+1}$ would surely be larger than the one that could be obtained solving the FHOCP at that time instant. So, also taking into account (20) defining $x_{c}\left(t_{k+1}\right)$ as

$$
x_{c}\left(t_{k+1}\right) \triangleq\left[\begin{array}{c}
\varphi\left(T, x_{c}\left(t_{k}\right), \mathbf{u}_{\left[t_{k}, t_{k+1} \mid t_{k}\right)}^{o} \mathbf{w}_{\left[t_{k}, t_{k+1}\right)}\right) \\
\mathbf{u}_{\left[t_{k+1}, t_{k+N} \mid t_{k}\right)}^{o}\left(t_{k+1}\right)
\end{array}\right]
$$

and $x_{c}\left(t_{k+N} \mid t_{k+1}\right)$ as in (43) (with $t=t_{k+1}$ ), and taking into account Point 6 in Assumption 3, it is possible to state that

$$
\begin{aligned}
& V\left(t_{k+1}, x_{c}\left(t_{k+1}\right), N\right) \\
& \leq J\left(x_{c}\left(t_{k+1}\right), \overline{\mathbf{u}}_{\left[t_{k+1}, t_{k+N} \mid t_{k+1}\right]}^{s}, N\right) \\
&= V\left(t_{k+1}^{-}, \varphi\left(T^{-}, x\left(t_{k}\right), \mathbf{u}_{\left[t_{k}, t_{k+1} \mid t_{k}\right.}^{o}, \mathbf{w}_{\left[t_{k}, t_{k+1}\right)}\right), N\right) \\
& \quad+\int_{0}^{T} l\left(\varphi\left(\zeta, x\left(t_{k+N} \mid t_{k+1}\right), \mathbf{u}_{\left[t_{k+N}, t_{k+N+1} \mid t_{k+1}\right)}^{s}, \mathbf{0}\right),\right. \\
&\left.\quad \mathbf{u}_{\left[t_{k+1}, t_{k+N+1} \mid t_{k+1}\right)}^{s}\right) d \zeta \\
&+V_{f}\left(\varphi\left(N T, x\left(t_{k+1}\right), \mathbf{u}_{\left[t_{k+1}, t_{k+N+1} \mid t_{k+1}\right)}^{s}, \mathbf{0}\right)\right) \\
&-V_{f}\left(\varphi\left(N T, x\left(t_{k}\right), \overline{\mathbf{u}}_{\left[t_{k}, t_{k+N} \mid t_{k}\right)}^{o}, \mathbf{0}\right)\right. \\
& \leq V\left(t_{k+1}^{-}, \varphi_{c}\left(T^{-}, x_{c}\left(t_{k}\right), \mathbf{w}_{\left[t_{k}, t_{k+1}\right)}\right), N\right)
\end{aligned}
$$

which proves that $V\left(t_{k}^{+}, x_{c}\left(t_{k}^{+}\right), N\right) \leq V\left(t_{k}^{-}, x_{c}\left(t_{k}^{-}\right), N\right), \forall k$. 
As for Point 4, it is necessary to guarantee that the region $\Theta_{\mathbf{w}}$ defined in (27) is contained in $\Xi$, which coincides with $\mathcal{X}^{M P C}$ in our case. $\Theta_{\mathbf{w}}$ depends on $\mathcal{W}^{\text {sup }}$ through functions and constants of which we are just able to compute very conservative upper-bounds. Then, the set $\mathcal{W}$ has to be such that the feasibility condition (21) holds, and that $\Theta_{\mathrm{w}} \subseteq \mathcal{X}^{M P C}$. Due to the conservativeness in the calculation of $\Theta_{\mathbf{w}}$, the last condition could be the most stringent. However, it is necessary just to give an estimation of the region where the state of the closed-loop system converges asymptotically. In order not to limit the applicability of the method only to extremely small uncertainties, we avoid to estimate $\Theta_{w}$, knowing that the actual region where the system will converge is included in $\mathcal{X}^{M P C}$, this latter being a robust positively invariant set.

Proof of Theorem 1: The application of the ISM to system (2), according to Lemma 2, leads to a system in form (12), i.e. system (2) with $w=B^{\perp} w_{\mathrm{U}}(t)$. Moreover, in order to apply the ISM inner loop, the control variable in the MPC control law is limited in the set $\overline{\mathcal{U}}^{R}$. Then, since Assumptions 1-4 are satisfied for system (2) with $w=B^{\perp} w_{\mathrm{U}}(t)$ and $\overline{\mathcal{U}}=\overline{\mathcal{U}}^{R}$, according to Lemma 4 the ISpS of the overall control scheme is proved.

\section{ACKNOWLEDGMENT}

The authors would like to thank Dr. E. Vitali, Department of Mathematics, University of Pavia, for the useful discussion about the proof of Theorem 2 .

\section{REFERENCES}

[1] M. Morari and J. H. Lee, "Model predictive control: Past, present and future," Comput. Chem. Eng., vol. 23, no. 4-5, pp. 667-682, 1999.

[2] D. Q. Mayne, J. B. Rawlings, C. V. Rao, and P. O. M. Scokaert, "Constrained model predictive control: Stability and optimality," Automatica, vol. 36, no. 6, pp. 789-814, 2000.

[3] S. J. Qin and T. A. Badgwell, "A survey of industrial model predictive control technology," Control Eng. Pract., vol. 11, no. 7, pp. 733-764, 2003.

[4] J. M. Maciejowski, Predictive Control With Constraints. Upper Saddle River, NJ: Pearson Education, 2002.

[5] E. F. Camacho and C. Bordons, Model Predictive Control. New York: Springer Verlag, 2004.

[6] J. B. Rawlings and D. Q. Mayne, Model Predictive Control: Theory and Design. Madison, WI: Nob Hill Publishing, 2009.

[7] , L. Magni, D. M. Raimondo, and F. Allgöwer, Eds., Nonlinear Model Predictive Control: Towards New Challenging Applications. New York: Springer-Verlag, 2009.

[8] D. Limon, T. Alamo, D. M. Raimondo, D. Muñoz de la Peña, J. M. Bravo, A. Ferramosca, and E. F. Camacho, "Input-to-state stability: A unifying framework for robust model predictive control," in Nonlinear Model Predictive Control: Towards New Challenging Applications, L. Magni, D. M. Raimondo, and F. Allgöwer, Eds. New York: SpringerVerlag, 2009, pp. 1-26.

[9] H. Chen, C. W. Scherer, and F. Allgöwer, "A game theoretical approach to nonlinear robust receding horizon control of constrained systems," in Proc. Amer. Control Conf., Albuquerque, NM, Jun. 1997, pp. 3073-3077.

[10] P. O. M. Scokaert and D. Q. Mayne, "Min-max feedback model predictive control for constrained linear systems," IEEE T. Autom. Control, vol. 43, no. 8, pp. 1136-1142, Aug. 1998.

[11] A. Bemporad, F. Borrelli, and M. Morari, "Min-max control of constrained uncertain discrete-time linear systems," IEEE T. Autom. Control, vol. 48, no. 9, pp. 1600-1606, Sep. 2003.

[12] F. Fontes and L. Magni, "Min-max model predictive control of nonlinear systems using discontinuous feedbacks," IEEE T. Autom. Control, vol. 48, no. 10, pp. 1750-1755, Oct. 2003.

[13] M. Diehl and J. Bjornberg, "Robust dynamic programming for min-max model predictive control of constrained uncertain systems," IEEE T. Autom. Control, vol. 49, no. 12, pp. 2253-2257, Dec. 2004.
[14] M. Lazar, D. Muñoz de la Peña, W. P. M. H. Heemels, and T. Alamo, "On input-to-state stability of min-max nonlinear model predictive control," Sys. Control Lett., vol. 57, no. 1, pp. 39-48, 2008.

[15] L. Chisci, J. A. Rossiter, and G. Zappa, "Systems with persistent disturbances: Predictive control with restricted constraints," Automatica, vol. 37, no. 7, pp. 1019-1028, 2001.

[16] D. Limon, T. Alamo, and E. F. Camacho, "Input-to-state stable MPC for constrained discrete-time nonlinear systems with bounded additive uncertainties," in Proc. Conf. Decision Control, Las Vegas, NV, Dec. 2002, pp. 4619-4624.

[17] S. V. Rakovic, A. R. Teel, D. Q. Mayne, and A. Astolfi, Simple Robust Control Invariant Tubes for Some Classes of Nonlinear Discrete Time Systems. San Diego, CA, Dec. 2006.

[18] G. Grimm, M. J. Messina, S. E. Tuna, and A. R. Teel, "Nominally robust model predictive control with state constraints," IEEE T. Autom. Control, vol. 52, no. 10, pp. 1856-1870, Oct. 2007.

[19] G. Pin, D. M. Raimondo, L. Magni, and T. Parisini, "Robust model predictive control of nonlinear systems with bounded and state-dependent uncertainties," IEEE T. Autom. Control, vol. 54, no. 7, pp. 1681-1687, Jul. 2009.

[20] V. I. Utkin, Sliding Mode in Control and Optimization. New York: Springer-Verlag, 1992.

[21] C. Edwards and S. Spurgeon, Sliding Mode Control: Theory and Applications. New York: Taylor \& Francis, 1998.

[22] W. Garcia-Gabin, D. Zambrano, and E. F. Camacho, "Sliding mode predictive control of a solar air conditioning plant," Control Eng. Pract., vol. 17, no. 6, pp. 652-663, 2009.

[23] K. R. Muske, H. Ashrafiuon, and M. Nikkhah, "A predictive and sliding mode cascade controller," in Proc. Amer. Control Conf., New York, Jul. 2007, pp. 4540-4545.

[24] V. I. Utkin and J. Shi, "Integral sliding mode in systems operating under uncertainty conditions," in Proc. Conf. Decision Control, Kobe, Japan, Dec. 1996, pp. 4591-4596.

[25] A. Poznyak, L. Fridman, and F. J. Bejarano, "Mini-max integral sliding-mode control for multimodel linear uncertain systems," IEEE T. Autom. Control, vol. 49, no. 1, pp. 97-102, Jan. 2004.

[26] M. Basin, J. Rodriguez, L. Fridman, and P. Acosta, "Integral sliding mode design for robust filtering and control of linear stochastic time-delay systems," Int. J. Robust Nonlin. Control, vol. 15, no. 9, pp. 407-421, 2005

[27] F. Castaños and L. Fridman, "Analysis and design of integral sliding manifolds for systems with unmatched perturbations," IEEE T. Autom. Control, vol. 51, no. 5, pp. 853-858, May 2006.

[28] F. A. C. C. Fontes, "A general framework to design stabilizing nonlinear model predictive controllers," Sys. Contr. Lett., vol. 42, no. 2, pp. 127-144, 2001.

[29] L. Magni and R. Scattolini, "Model predictive control of continuous-time nonlinear systems with piecewise constant control," IEEE T. Autom. Control, vol. 49, no. 6, pp. 900-906, Jun. 2004.

[30] L. Grune, D. Nesic, and J. Pannek, "Model predictive control for nonlinear sampled-data systems," in Nonlinear Model Predictive Control: Towards New Challenging Applications, R. Findeisen, F. Allgöwer, and L. Biegler, Eds. New York: Springer-Verlag, 2007, pp. 105-113.

[31] L. Magni, D. M. Raimondo, and R. Scattolini, "Regional input-to-state stability for nonlinear model predictive control," IEEE T. Autom. Control, vol. 51, no. 9, pp. 1548-1553, Sep. 2006.

[32] D. M. Raimondo, D. Limon, M. Lazar, L. Magni, and E. F. Camacho, "Min-max model predictive control of nonlinear systems: A unifying overview on stability," Eur. J. Control, vol. 15, no. 1, pp. 5-21, 2009.

[33] M. Rubagotti, D. M. Raimondo, A. Ferrara, and L. Magni, Robust Model Predictive Control of Continuous-Time Sampled-Data Nonlinear Systems With Integral Sliding Mode. Budapest, Hungary, Aug. 2009.

[34] W. J. Cao and J. X. Xu, "Nonlinear integral-type sliding surface for both matched and unmatched uncertain systems," IEEE T. Autom. Control, vol. 49, no. 8, pp. 1355-1360, Aug. 2004.

[35] L. Magni, G. De Nicolao, Scattolini, and F. R. Allgöwer, "Robust model predictive control for nonlinear discrete-time systems," Int. J. Robust Nonlin. Control, vol. 13, no. 3-4, pp. 229-246, 2003.

[36] G. Bartolini, A. Ferrara, and E. Usai, "Chattering avoidance by secondorder sliding mode control," IEEE T. Autom. Control, vol. 43, no. 2, pp. 241-246, Feb. 1998

[37] L. Fridman, "An averaging approach to chattering," IEEE T. Autom. Control, vol. 46, no. 8, pp. 1260-1265, Aug. 2001.

[38] L. Fridman, "Chattering analysis in sliding mode systems with inertial sensors," Int. J. Control, vol. 9, no. 10, pp. 906-912, 2003.

[39] A. Levant, "Chattering analysis," in Proc. Eur. Control Conf., Kos, Greece, Jul. 2007, pp. 3195-3202. 
[40] E. D. Sontag and Y. Wang, "New characterizations of input-to statestability," IEEE T. Autom. Control, vol. 41, no. 9, pp. 1283-1294, Sep. 1996.

[41] D. Limon, T. Alamo, F. Salas, and E. F. Camacho, "Input to state stability of min-max MPC controllers for nonlinear systems with bounded uncertainties," Automatica, vol. 42, no. 5, pp. 797-803, 2006.

[42] J. Y. Lin, E. D. Sontag, and Y. Wang, "A smooth converse Lyapunov theorem for robust stability," SIAM J Control Optim., vol. 34, no. 1, pp. 124-160, 1996.

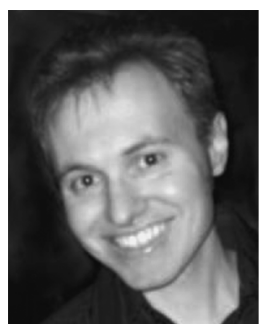

Matteo Rubagotti (S'07) was born in Voghera (PV), Italy, in 1982. He received the "Laurea" and "Laurea Specialistica" degrees (with highest honors) in computer engineering, and the Ph.D. degree in electronic, computer science and electrical engineering from the University of Pavia, Italy, in 2004, 2006, and 2010, respectively.

From 2008 to 2009, he was a Visiting Scholar at the Ohio State University Center for Automotive Research, Columbus, OH. From July to August 2009 he was an Academic Guest at the Automatic Control Laboratory, ETH Zürich, Switzerland. Since January 2010, he is a Post-Doc with the Department of Mechanical and Structural Engineering, University of Trento, Italy. His research is mainly focused on analysis and controller synthesis of uncertain nonlinear and piecewise-affine systems (in particular, using sliding mode and model predictive control techniques), and on mobile robotics.

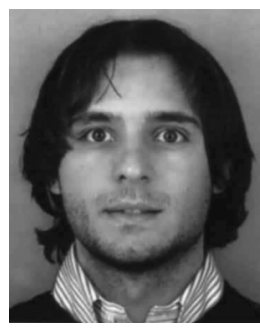

Davide Martino Raimondo was born in Pavia, Italy, in 1981. He received the Laurea degree (with highest honors) in computer engineering and the Ph.D. degree in electronic, computer and electrical engineering from the University of Pavia, Italy, in 2005 and 2009, respectively.

From November 2006 to May 2007, he was with the Escuela Superior de Ingenieros, Universidad de Sevilla, Sevilla, Spain. Since January 2009 he is working as post-doc at Automatic Control Laboratory, ETH Zürich, Switzerland. He is the author or coauthor of more than 30 papers published in refereed journals, edited books, and refereed conference proceedings. His current research interests include nonlinear control, model predictive control, networked control, robust control, input-to-state stability, and glycemia control in diabetic subjects.

Dr. Raimondo co-organized the NMPC Workshop on Assessment and Future Direction, Pavia, Italy, in 2008. He was also co-organizer of the invited sessions Nonlinear Model Predictive Control, for IFAC NOLCOS 2010, and New Developments in Nonlinear Model Predictive Control, for IFAC NOLCOS 2007. He served as an Associate Editor of IFAC NOLCOS 2010.

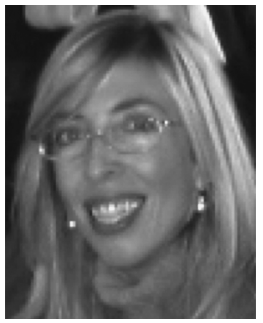

Antonella Ferrara (S'86-M'88-SM'03) was born in Genova, Italy. She received the Laurea degree in electronic engineering and the Ph.D. degree in computer science and electronics from the University of Genova, Genova, Italy, in 1987 and 1992, respectively.

She has been Assistant Professor at the University of Genova since 1992. In November 1998 she became Associate Professor of Automatic Control at the University of Pavia. Since January 2005 she is Full Professor of Automatic Control in the Department of Computer Engineering and Systems Science, University of Pavia. Her research activities are mainly in the area of sliding mode control, with application to automotive control, process control, and robotics. She has authored or coauthored more than 230 papers, including more than 70 international journal papers.

Dr. Ferrara was an Associate Editor of the IEEE TRANSACTIONS ON CONTROL Systems TeChNOLOGY and, at present, she is Associate Editor of the IEEE TRANSACTIONS ON AUTOMATIC CONTROL. She is a member of the IEEE Technical Committee on Variable Structure and Sliding Mode Control, a member of the IEEE Robotics and Automations Technical Committee on Autonomous Ground Vehicles and Intelligent Transportation Systems, and a member of the IFAC Technical Committee on Transportation Systems.

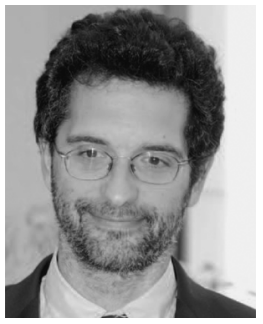

Lalo Magni was born in Bormio (SO), Italy, in 1971. He received the M.S. degree (with highest honors) in computer engineering and the $\mathrm{Ph} . \mathrm{D}$. degree in electronic and computer engineering from the University of Pavia, Pavia, Italy, in 1994 and 1998, respectively.

From 1999 to 2004, he was Assistant Professor at the University of Pavia where he has been Associate Professor since 2005. From 1996 to 1997 and in 1998 he was at CESAME, Universit Catholique de Louvain, Louvain La Neuve (Belgium). From October to November 1997, he was at the University of Twente with the System and Control Group in the Faculty of Applied Mathematics. In 2003, he was a Plenary Speaker at the Second IFAC Conference "Control Systems Design" (CSD'03). In 2005, he was Keynote speaker at the NMPC Workshop on Assessment and Future Direction. His current research interests include nonlinear control, predictive control, robust control, process control and glucose concentration control in subjects with diabetes. His research is witnessed by more than 40 papers published in the main international journals. In 2003, he was Guest Editor of the Special Issue 'Control of nonlinear systems with Model Predictive Control' in the International Journal of Robust and Nonlinear Control.

Dr. Magni was an Associate Editor of the IEEE TRANSACTIONS ON Automatic CONTROL. He is Associate Editor of Automatica. He organized the NMPC Workshop on Assessment and Future Direction in September 2008 in Pavia. 